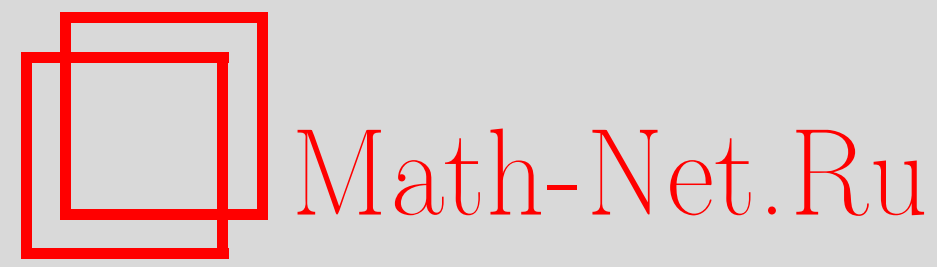

S. Fortini, L. Ladelli, E. Regazzini, A central limit problem for partially exchangeable random variables, Теория вероятн. и ее примен., 1996, том 41, выпуск 2, 353-379

DOI: https://doi.org/10.4213/tvp2943

Использование Общероссийского математического портала Math-Net.Ru подразумевает, что вы прочитали и согласны с пользовательским соглашением http://www.mathnet.ru/rus/agreement

Параметры загрузки:

IP : 52.6 .47 .48

26 апреля 2023 г., 15:02:48

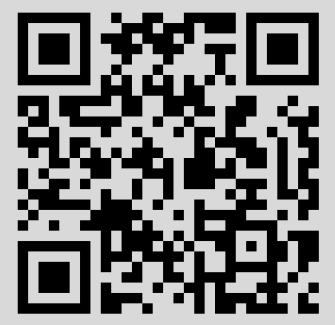




\section{A CENTRAL LIMIT PROBLEM FOR PARTIALIY EXCHANGEABLE RANDOM VARIABLES ${ }^{1)}$}

В настоящей статье рассматривается центральная предельная проблема для $\left(\left(S_{1 n}, S_{2 n}, \ldots\right)\right)_{n}$, когда $S_{i n}=\sum_{j=1}^{n} \xi_{i j}^{(n)}(i=1,2, \ldots)$, а $\left\{\xi_{i j}^{(n)}: i=1,2, \ldots ; j=1, \ldots, n\right\}$ для каждого $n$ есть матрица частично перестановочных случайных величин. Показано, что при определенных условиях «пренебрежимости» класс предельных законов совпадает с классом всех перестановочных законов, которые представимы в виде смесей безгранично делимых распределений. Более того, даны необходимые и достаточные условия сходимости $\mathrm{K}$ любому заданному элементу из этого класса. Обсуждаются критерии для трех замечательных типов предельных законов (смесь гауссовских, пуассоновских и вырожденных распределений). Доказано также, что в случае, когда $\xi_{i j}^{(n)}=X_{i j} / a_{n},\left(a_{n} \rightarrow+\infty\right)$, а величины $X_{i j}(i, j=1,2, \ldots)$ перестановочны, класс предельных законов можно охарактеризовать в терминах смесей устойчивых законов. Наконец, мы показываем, что некоторые хорошо известные основные предельные теоремы могут быть получены как следствия результатов, доказанных в настоящей работе.

Ключевые слова и фразы: центральная предельная проблема, теорема представления де Финетти, безгранично делимые законы и их смеси, частично перестановочные случайные величины, устойчивые законы и их смеси, теорема представления Скорохода.

\section{Introduction}

For every $n=1,2, \ldots$, let $\left\{\xi_{i j}^{(n)}: i=1,2, \ldots ; j=1, \ldots n\right\}$ be an array (with infinitely many rows and $n$ columns) of partially exchangeable random variables, in the sense that the probability distribution of every finite subset of the array is invariant with respect to all finite permutations $\sigma_{1}, \ldots, \sigma_{n}$ acting on the first, $\ldots, n$th column, respectively. Then, for the $n$th array

*CNR-IAMI, Università degli Studi, Università «L. Bocconi». Milano.

1) Research partially supported by MURST (40\%, 1993-1994, «Inferenza statistica: basi probabilistiche e sviluppi metodologici» and «Processi stocastici e calcolo stocastico») and by Università «L. Bocconi» (fondi di ricerca IMQ, 1993). 
$(n=1,2, \ldots)$, consider the sequence of row sums

$$
S_{i n}=\sum_{j=1}^{n} \xi_{i j}^{(n)}, \quad i=1,2, \ldots
$$

The present paper deals with the following central limit problem:

- Which is the class of limit laws of $\left(\left(S_{1 n}, S_{2 n}, \ldots\right)\right)_{n}$ as $n \rightarrow \infty$ ? law?

- Under which conditions does $\left(\left(S_{1 n}, S_{2 n}, \ldots\right)\right)_{n}$ converge to a specified

The study of this problem is motivated by the need for reasonable justifications for the adoption of suitable models in the Bayesian analysis of those situations in which observable random quantities are viewed as sums of small effects of a great number of factors.

Section 4 in the present paper shows that, under suitable «negligibility» conditions, the class of limiting laws coincides with that of all exchangeable laws which are presentable as linear convex combinations of infinitely divisible distributions. In the same section, necessary and sufficient conditions for convergence to any specified element of that class are provided. Section 5 includes criteria for three remarkable limit types: mixture of Gaussian, Poisson, degenerate probability distributions. Section 6 considers the array of random variables

$$
\xi_{i j}^{(n)}=\frac{X_{i j}}{a_{n}} \quad a_{n}>0, \quad a_{n} \rightarrow+\infty,
$$

when the $X_{i j}$ 's $(i, j=1,2, \ldots)$ are assumed to be exchangeable. Under this assumption, the class of limiting laws can be characterized in terms of mixtures of stable laws.

The arguments used to prove the previous statements rely substantially on de Finetti's representation theorem for partially exchangeable random variables (see Section 1) and on the Skorokhod method for representing weakly convergent sequences of probability measures by sequences of random elements which converge almost surely; see Section 3. In fact, the Skorokhod method is used to represent the conditional convolutions which appear in de Finetti's representation of the law of $\left(S_{1 n}, S_{2 n}, \ldots\right)$ and, consequently, the method at issue allows one to resort to the central limit theorem for independent random variables.

Finally, in Section 7, it is shown that a few basic, well-known central limit theorems for sequences of exchangeable random variables (reviewed in [17]) can be obtained as simple corollaries of the main statements included in Sections 5-6 of the present paper. In particular, the deduction from the same statements of a central limit theorem for exchangeable random variables without moments, originally proved by Klass and Teicher [13], is worth noticing; see Subsection 7.4.

A unified method of deriving weak limit results (giving convergence to Gaussian and Poisson laws) for arrays of (partially) exchangeable random 
variables, based on known results for arrays of martingale differences, is explained by Eagleson in [4]. The connection between the exchangeable arrays and the martingale results is made by introducing conditional expectations, given suitable families of $\sigma$-fields. General limit theorems about sequences of conditional expectations, given some specific system of $\sigma$-fields, are proved in [14] and [19]. However, these papers do not provide information about the choice of suitable $\sigma$-fields in order to obtain general, explicit answers to the central limit problem for (partially) exchangeable summands. Going back to the Eagleson approach, it is worth noticing that this is applicable whether the number of the rows in the array be infinite or finite. On the other hand, the same approach generally yields sufficient - but not necessary conditions for convergence.

\section{Preliminaries and notation}

The definition of infinitely divisible (i.d.) probability distribution function (p.d.f.) was introduced by de Finetti in connection with his pioneer investigations into processes with stationary independent increments; see [6]. A p.d.f. is called i.d. if for every integer $n$ there exists a p.d.f. $F_{n}$ whose $n$-fold convolution $\left(F_{n}\right)^{n \star}$ coincides with $F$ or, equivalently, if its FourierStieltjes transform $\varphi$ is the $n$th power of a characteristic function (c.f.) $\varphi_{n}$ for every $n$.

De Finetti [7] proved that the class of i.d. laws coincides with the class of distribution limits of finite convolutions of distributions of the Poisson type. Kolmogorov [12] and Lévy [15] provided more useful representations. Lévy's representation is general, but somewhat convoluted. Consequently, one generally refers to the following characterization due to [11]:

$\phi$ is the c.f. of an i.d. law if, and only if,

$$
\phi(t)=\exp \left\{i \alpha t+\int_{\mathbf{R}}\left(e^{i t x}-1-\frac{i t x}{1+x^{2}}\right) \frac{1+x^{2}}{x^{2}} d G(x)\right\}, \quad t \in \mathbf{R},
$$

where the integrand is defined at $x=0$ by continuity $\left(-t^{2} / 2\right), \alpha$ is a real number and $G$ - the Lévy-Khintchine spectral function - is a nondecreasing, right continuous function on the extended real line $\overline{\mathbf{R}}$ with $G(-\infty)=0$, $\lim _{x \rightarrow+\infty} G(x)=G(+\infty)<+\infty$. $\phi$ uniquely determines $(\alpha, G)$.

Usually the Lévy-Khintchine spectral function is defined on $\mathbf{R}$. Here one considers $G$ on $\overline{\mathbf{R}}$ because of technical reasons arising in Section 3 .

In the present paper one considers mixtures of i.d. c.f.'s with respect to (w.r.t.) the parameter $(\alpha, G)$ and then, in order to define the corresponding mixing probability measures, one introduces the following notation:

$\mathbf{E}$ for a metric space;

$B(\mathbf{E})$ for the Borel class of $\mathbf{E}$;

$\mathbf{M}(\mathbf{E})$ for the class of all finite measures on $B(\mathbf{E})$;

$\mathbf{P}(\mathbf{E})$ for the set of all probability measures on $\mathcal{B}(\mathbf{E})$; 
$\mathcal{D}$ for the class of all bounded distribution functions (d.f.) $D$ on the extended real line $\overline{\mathbf{R}}$.

Moreover, given $\lambda$ in $\mathbf{M}(\overline{\mathbf{R}}), D_{\lambda}$ will denote the corresponding d.f. and, given $D$ in $\mathcal{D}, \lambda_{D}$ will denote the corresponding Lebesgue-Stieltjes measure. Clearly, the spectral function in (1.1) belongs to the class $\mathcal{D}_{0}$ of the d.f.'s $D$ in $\mathcal{D}$ such that $D(-\infty)=0$ and $\lim _{x \rightarrow+\infty} D(x)=D(+\infty)$.

A sequence $\left(\lambda_{n}\right)_{n}$ in $\mathbf{M}(\mathbf{E})$ is called completely convergent to $\lambda$ in $\mathbf{M}(\mathbf{E})$ if

$$
\int_{\mathbf{E}} f d \lambda_{n} \rightarrow \int_{\mathbf{E}} f d \lambda
$$

for every $f$ in the class $\mathbf{C}_{b}(\mathbf{E})$ of bounded continuous real-valued functions on $\mathbf{E}$. In particular, if $\lambda_{n}(n=1,2, \ldots)$ and $\lambda$ belong to $\mathbf{P}(\mathbf{E})$, then complete convergence is usually called weak convergence of probability measures and one writes $\lambda_{n} \Rightarrow \lambda$. Moreover, if $X_{n}(n=1,2, \ldots)$ and $X$ are random elements from a measurable space $(\Omega, \mathcal{F})$ into $(\mathbf{E}, \mathcal{B}(\mathbf{E}))$, and if the probability distribution (p.d.) of $X_{n}$ converges weakly to the p.d. of $X$, then $\left(X_{n}\right)_{n}$ is said to be convergent in law to $X$ and one writes $X_{n} \stackrel{\ell}{\longrightarrow} X$.

A sequence $\left(\mu_{n}\right)_{n}$ of probability measures is called relatively compact if each subsequence has a subsequence converging weakly to a probability measure. If $\mathbf{E}$ is Polish and $\left(\mu_{n}\right)_{n}$ is relatively compact, then $\left(\mu_{n}\right)_{n}$ is tight, that is: for every $\varepsilon>0$ there exists a compact set $K_{\varepsilon} \subset \mathrm{E}$ such that $\mu_{n}\left(K_{\varepsilon}\right)>$ $1-\varepsilon$ for all $n$. If $\mathbf{E}$ is any metric space and if $\left(\mu_{n}\right)_{n}$ is tight, then $\left(\mu_{n}\right)_{n}$ is relatively compact. These propositions will be referred to as Prokhorov's theorem; see $[1$, p. 37].

Finally, we quote the notion of partially exchangeable real-valued random variables (r.v.'s). For each positive integer $n$, let

$$
\xi^{(n)}=\left\{\begin{array}{cccc}
\xi_{11}^{(n)} & \xi_{12}^{(n)} & \cdots & \xi_{1 n}^{(n)} \\
\xi_{21}^{(n)} & \xi_{22}^{(n)} & \cdots & \xi_{2 n}^{(n)} \\
\cdots & \cdots & \cdots & \cdots \\
\xi_{i 1}^{(n)} & \xi_{i 2}^{(n)} & \cdots & \xi_{i n}^{(n)} \\
\cdots & \cdots & \cdots & \cdots
\end{array}\right.
$$

be an array of real r.v.'s on the probability space $(\Omega, \mathcal{F}, P)$, with $n$ columns and an infinity of rows. According to the definition mentioned in Introduction, the elements of (1.3) are said to be partially exchangeable (or (1.3) is said to be partially exchangeable) if, for all finite permutations $\sigma_{1}, \sigma_{2}, \ldots, \sigma_{n}$, $\xi^{(n)}$ and

$$
\xi_{\sigma}^{(n)}:=\left\{\begin{array}{llll}
\xi_{\sigma_{1}(1) 1}^{(n)} & \xi_{\sigma_{2}(1) 2}^{(n)} & \cdots & \xi_{\sigma_{n}(1) n}^{(n)} \\
\xi_{\sigma_{1}(2) 1}^{(n)} & \xi_{\sigma_{2}(2) 2}^{(n)} & \cdots & \xi_{\sigma_{n}(2) n}^{(n)} \\
\cdots \cdots & \cdots & \cdots & (\cdots \\
\xi_{\sigma_{1}(i) 1}^{(n)} & \xi_{\sigma_{2}(i) 2}^{(n)} & \cdots & \xi_{\sigma_{n}(i) n}^{(n)} \\
\cdots & \cdots & \cdots & \cdots
\end{array}\right.
$$


have the same p.d. This is the notion of partial exchangeability originally proposed by de Finetti [8]. Clearly, if $\xi^{(n)}$ is partially exchangeable, then each column is exchangeable.

By the same argument used to prove de Finetti's representation theorem for exchangeable r.v.'s (cf. (3.1) in [20] and Subsection 2.2 in [21]), one obtains that the elements of $\xi^{(n)}$ are partially exchangeable if, and only if, there exist a $\sigma$-algebra $\mathcal{G}^{(n)} \subset \mathcal{F}$ and a random vector $\left(F_{1 n}(\cdot, \omega), \ldots, F_{n n}(\cdot, \omega)\right)$ of random p.d.f.'s in $\mathcal{D}_{0}$ such that

$$
\begin{gathered}
P\left(\bigcap_{i=1}^{m}\left\{\xi_{i 1}^{(n)} \leqslant x_{i 1}, \ldots, \xi_{i n}^{(n)} \leqslant x_{i n}\right\} \mid \mathcal{G}^{(n)}\right) \\
\quad=\prod_{i=1}^{m} \prod_{k=1}^{n} F_{k n}\left(x_{i k}, \omega\right) \quad(P \text {-a.s. })
\end{gathered}
$$

for every $\left(x_{i 1}, \ldots, x_{i n}\right)$ in $\mathbf{R}^{n}, i=1, \ldots, m, m=1,2, \ldots$

It should be noted that the random vector $\left(F_{1 n}, \ldots, F_{n n}\right)$ is unique $P$ a.s.; cf. (2.15) in [20]. Clearly, $\xi^{(n)}$ is exchangeable if, and only if, $F_{k n}=F_{1 n}$ $P$-a.s. $(k=2, \ldots, n)$.

\section{A central limit problem for partially exchangeable r.v.'s}

Define $\left(\xi^{(n)}\right)_{n}$ to be a sequence of arrays of r.v.'s as in (1.3) and assume $\left(\mathrm{H}_{1}\right)$ For each $n$, the elements of $\xi^{(n)}$ are partially exchangeable.

Hence, after putting

$$
S_{i n}=\sum_{k=1}^{n} \xi_{i k}^{(n)}, \quad i=1,2, \ldots, \quad n=1,2, \ldots,
$$

the present paper deals with the central limit problem explained in Introduction. This setting can be motivated by paraphrasing Example (2.5) in [5] dealing with the p.d. of a numerical characteristic of some mass produced article, when such a characteristic is viewed as the sum of a real number and of a cumulative error due to a great number, say $n$, of factors, whose effects $\xi_{i k}^{(n)}$ on the $i$ th article produced are negligible $(i=1,2, \ldots ; k=1, \ldots, n)$. If articles are produced under similar environmental conditions and if the $n$ factors turn out to be distinguishable, then $\left(\mathrm{H}_{1}\right)$ provides a suitable starting point for modeling the p.d. of $\left(S_{1 n}, S_{2 n} \ldots\right)$. As far as the magnitude of each $\xi_{i k}^{(n)}$ is concerned, one has to make precise the concept of negligibility. Taking advantage of $\left(\mathrm{H}_{1}\right)$ and, consequently, of (1.4), one introduces the following r.v.'s

$$
M_{n}(\varepsilon)=M_{n}(\varepsilon, \omega)=\max _{1 \leqslant i \leqslant n} \lambda_{F_{\text {in }}}\left([-\varepsilon, \varepsilon]^{c}\right), \quad \varepsilon>0, \quad n=1,2, \ldots,
$$

and one can consider the elements of $\left(\xi^{(n)}\right)_{n}$ as negligible if

$$
M_{n}(\varepsilon) \stackrel{P}{\longrightarrow} 0, \quad \varepsilon>0, \quad n \rightarrow+\infty,
$$


where $\stackrel{P}{\rightarrow}$ denotes convergence in probability.

When the elements of $\left(\xi^{(n)}\right)_{n}$ satisfy $\left(\mathrm{H}_{1}\right)$ and $\left(\mathrm{H}_{2}\right)$, then they are called conditionally uniformly asymptotically negligible (conditionally u.a.n.).

If $\left(\mathrm{H}_{2}\right)$ holds, then for each $\varepsilon>0$ one can select a subsequence $n_{k}(\varepsilon)$ of (n) such that

$$
M_{n_{k}(\varepsilon)}(\varepsilon) \longrightarrow 0, \quad k \rightarrow+\infty, \quad(P \text {-a.s. })
$$

and there is $\Omega_{1} \subset \Omega$ with $P\left(\Omega_{1}\right)=1$ such that

$$
M_{n_{k}(1 / m)}\left(\frac{1}{m}\right) \longrightarrow 0, \quad k \rightarrow+\infty,
$$

for every $m=1,2, \ldots$ and for every $\omega \in \Omega_{1}$. Then, by the diagonal method, one can fix a strictly increasing sequence $\gamma_{1}, \gamma_{2} \ldots$ of integers for which

$$
\lim _{k \rightarrow+\infty} M_{\gamma_{k}}\left(\frac{1}{m}\right)=0, \quad m=1,2, \ldots, \quad \omega \in \Omega_{1},
$$

and, therefore,

$$
\lim _{k \rightarrow+\infty} M_{\gamma_{k}}(\varepsilon)=0, \quad \varepsilon>0, \quad \omega \in \Omega_{1} .
$$

R e m a r k. Condition $\left(\mathrm{H}_{2}\right)$ and, consequently, (2.1) are implied by

$$
\max _{1 \leqslant k \leqslant n} P\left(\left|\xi_{1 k}^{(n)}\right|>\varepsilon\right)=o\left(\frac{1}{d(n)}\right), \quad n \rightarrow+\infty, \quad \varepsilon>0,
$$

provided that $d(n)$ denotes the number of distinct elements in $\left(F_{1 n}, \ldots, F_{n n}\right)$. Indeed, for every $x>0$ :

$$
P\left(M_{n}(\varepsilon)>x\right) \leqslant \sum_{i}^{(*)} \frac{E\left(\lambda_{F_{i n}}\left([-\varepsilon, \varepsilon]^{c}\right)\right)}{x}=o(1), \quad n \rightarrow+\infty, \quad \varepsilon>0,
$$

where the sum $\sum_{i}^{(*)}$ is extended to the $i$ 's corresponding to distinct $F_{i n}$ 's.

Although more restrictive than $\left(\mathrm{H}_{2}\right)$, condition $\left(\mathrm{K}_{2}\right)$ may be of interest for statistical applications.

In order to state conditions under which $\left(\left(S_{1 n}, S_{2 n}, \ldots\right)\right)_{n}$ converges to a specified law, define, for every $\omega$ in $\Omega$ and $r>0$,

$$
\begin{aligned}
\alpha_{n}^{(r)}(\omega) & =\sum_{k=1}^{n}\left\{m_{k n}^{(r)}(\omega)+\int_{\mathbf{R}} \frac{x}{1+x^{2}} d \bar{F}_{k n}^{(r)}(x, \omega)\right\} \\
\psi_{n}^{(r)}(x, \omega) & =\sum_{k=1}^{n} \int_{(-\infty, x]} \frac{y^{2}}{1+y^{2}} d \bar{F}_{k n}^{(r)}(y, \omega)
\end{aligned}
$$

where

$$
m_{k n}^{(r)}(\omega)=\int_{|x|<r} x d F_{k n}(x, \omega), \quad \bar{F}_{k n}^{(r)}(x, \omega)=F_{k n}\left(x+m_{k n}^{(r)}(\omega), \omega\right) .
$$


For each fixed $r>0$ and positive integer $n$, let $\nu_{n}^{(r)}$ be the probability measure on $\mathcal{B}\left(\mathbf{R} \times \mathbf{M}_{0}(\overline{\mathbf{R}})\right)$ determined by

$$
\nu_{n}^{(r)}(A \times B)=P\left(\alpha_{n}^{(r)} \in A, \lambda_{\psi_{n}^{(r)}} \in B\right)
$$

for every $A$ in $\mathcal{B}(\mathbf{R})$ and $B$ in $\mathcal{B}\left(\mathbf{M}_{0}(\overline{\mathbf{R}})\right)$, where $\mathbf{M}_{0}(\overline{\mathbf{R}})=\{\lambda \in \mathbf{M}(\overline{\mathbf{R}})$ : $\lambda\{-\infty,+\infty\}=0\}$.

\section{A preliminary result}

This section deals with the Skorokhod representation, mentioned in Introduction, concerning the sequences $\left(W_{n}\right)_{n}$ and $\left(W_{n}^{(1)}\right)_{n}$, defined by

$$
\begin{aligned}
W_{n} & =\left(\alpha_{n}^{(r)}, \lambda_{\psi_{n}}^{(r)}, \lambda_{\Gamma_{n}}, \lambda_{F_{1 n}}, \ldots, \lambda_{F_{n n}}, \delta_{0}, \delta_{0}, \ldots, M_{n}\left(\frac{1}{2}\right), M_{n}\left(\frac{1}{3}\right), \ldots\right), \\
W_{n}^{(1)} & =\left(\lambda_{\Gamma_{n}}, \lambda_{F_{1 n}}, \ldots, \lambda_{F_{n n}}, \delta_{0}, \delta_{0}, \ldots, M_{n}\left(\frac{1}{2}\right), M_{n}\left(\frac{1}{3}\right), \ldots\right),
\end{aligned}
$$

where $\delta_{a}$ is the unit mass at $a$ and $\Gamma_{n}$ denotes the convolution $F_{1 n} * \cdots * F_{n n}$. Here, $W_{n}$ is viewed as a random element from $(\Omega, \mathcal{F})$ into $(\mathcal{S}, \mathcal{B}(\mathcal{S}))$, with $\mathcal{S}=\mathbf{R} \times \mathbf{M}_{0}(\overline{\mathbf{R}}) \times \mathbf{P}(\overline{\mathbf{R}})^{\infty} \times \overline{\mathbf{R}}^{\infty}\left(\mathbf{M}_{0}(\overline{\mathbf{R}})\right.$ and $\mathbf{P}(\overline{\mathbf{R}})$ in the complete convergence topology) whilst $W_{n}^{(1)}$ is viewed as a random element from $(\Omega, \mathcal{F})$ into $\left(\mathcal{S}^{(1)}, \mathcal{B}\left(\mathcal{S}^{(1)}\right)\right)$ with $\mathcal{S}^{(1)}=\mathbf{P}(\overline{\mathbf{R}})^{\infty} \times \overline{\mathbf{R}}^{\infty}$. In the following, $Q_{n}$ and $Q_{n}^{(1)}$ will denote the p.d.'s of $W_{n}$ and $W_{n}^{(1)}$, respectively.

Lemma 1. Assume that $\left(\mathrm{H}_{2}\right)$ holds.

(i) If, for a fixed positive $r,\left(\nu_{n}^{(r)}\right)_{n}$ converges weakly, then each subsequence of $\left(Q_{n}\right)_{n}$ includes a subsequence $\left(Q_{n^{\prime}}\right)_{n^{\prime}}$ such that $Q_{n^{\prime}} \Rightarrow Q$ and $Q\left(\mathbf{R} \times \mathbf{M}_{0}(\overline{\mathbf{R}}) \times \mathbf{P}(\overline{\mathbf{R}}) \times\left\{\delta_{0}\right\}^{\infty} \times\{0\}^{\infty}\right)=1$.

(ii) If $\left(S_{1 n}\right)_{n}$ converges in law, then each subsequence of $\left(Q_{n}^{(1)}\right)_{n}$ includes a subsequence $\left(Q_{n^{\prime \prime}}^{(1)}\right)_{n^{\prime \prime}}$ such that $Q_{n^{\prime \prime}}^{(1)} \Rightarrow Q^{(1)}$ and $Q^{(1)}\left(\mathbf{P}_{0}(\overline{\mathbf{R}}) \times\left\{\delta_{0}\right\}^{\infty} \times\right.$ $\left.\{0\}^{\infty}\right)=1$, where $\mathbf{P}_{0}(\overline{\mathbf{R}})=\{\lambda \in \mathbf{P}(\overline{\mathbf{R}}): \lambda(\{-\infty,+\infty\})=0\}$.

P r o of. (i) Since $\left(\nu_{n}^{(r)}\right)_{n}$ converges weakly and $\mathbf{P}(\overline{\mathbf{R}})^{\infty} \times \overline{\mathbf{R}}^{\infty}$ is compact, $\left(Q_{n}\right)_{n}$ turns out to be relatively compact. Then, in view of $\left(\mathrm{H}_{2}\right)$, we can chose a subsequence $\left(n^{\prime}\right)$ such that both $\left(Q_{n^{\prime}}\right)_{n^{\prime}}$ converges and

$$
\lim _{n^{\prime} \rightarrow+\infty} M_{n^{\prime}}(\varepsilon)=0
$$

for every $\varepsilon>0$ and $\omega \in \Omega_{1}$ with $P\left(\Omega_{1}\right)=1$. In its turn, (3.1) implies that $\left(M_{n^{\prime}}\left(\frac{1}{2}\right), M_{n^{\prime}}\left(\frac{1}{3}\right), \ldots\right)$ converges to $(0,0, \ldots)$ for every $\omega$ in $\Omega_{1}$ and $\lambda_{F_{k n^{\prime}}} \Rightarrow \delta_{0}$ $\left(n^{\prime} \rightarrow+\infty, k=1,2, \ldots\right)$. Therefore the thesis follows from the convergence of $\nu_{n}^{(r)}$.

(ii) $\mathbf{P}(\overline{\mathbf{R}})$ is compact in view of Theorem VIII.5(d) in [3]. Hence the sequence of probability measures defined by

$$
Q_{n}^{(2)}(A)=P\left(\lambda_{\Gamma_{n}} \in A\right), \quad A \in \mathcal{B}(\mathbf{P}(\overline{\mathbf{R}})), \quad n=1,2, \ldots,
$$


is tight and, therefore, it includes a convergent subsequence $\left(Q_{n^{\prime}}^{(2)}\right)_{n^{\prime}}$. Now from the convergence of $\left(S_{1 n}\right)_{n}$, for each $m=1,2, \ldots$, there is $K_{m}$ in $\mathbf{R}$ with $K_{m} \uparrow+\infty$ as $m \rightarrow+\infty$ and

$$
\varlimsup_{n^{\prime}} P\left(\left|S_{1 n^{\prime}}\right|>K_{m}\right) \leqslant \frac{1}{m} .
$$

On the other hand, if $D_{n^{\prime}}^{(m)}(\eta)=Q_{n^{\prime}}^{(2)}\left(\left\{\mu \in \mathbf{P}(\overline{\mathbf{R}}): \mu\left(\overline{\mathbf{R}} \backslash\left[-K_{m}, K_{m}\right]\right) \leqslant \eta\right\}\right)$ for every $\eta \in(0,1)$

$$
\begin{aligned}
P\left(\left|S_{1 n^{\prime}}\right|>K_{m}\right) & \geqslant \eta\left\{1-D_{n^{\prime}}^{(m)}(\eta)\right\}, \\
\varlimsup_{n^{\prime}}\left(1-D_{n^{\prime}}^{(m)}(\eta)\right) & \leqslant \frac{1}{m \eta} .
\end{aligned}
$$

Since the set

$$
C_{\eta}^{(m)}=\left\{\mu \in \mathbf{P}(\overline{\mathbf{R}}): \mu\left(\overline{\mathbf{R}} \backslash\left[-K_{m}, K_{m}\right]\right) \leqslant \eta\right\}
$$

is closed and $\bigcap_{m} C_{\eta}^{(m)}$ is included in $C_{\eta}^{(\infty)}=\{\mu \in \mathbf{P}(\overline{\mathbf{R}}): \mu\{-\infty,+\infty\} \leqslant \eta\}$, from Theorem 2.1 (iii) in [1],

$$
Q^{(2)}\left(C_{\eta}^{(m)}\right) \geqslant \varlimsup_{n^{\prime}} Q_{n^{\prime}}^{(2)}\left(C_{\eta}^{(m)}\right)
$$

and, by letting $m \rightarrow+\infty$,

$$
Q^{(2)}\left(C_{\eta}^{(\infty)}\right) \geqslant 1 \quad \forall \eta \in(0,1) .
$$

This shows that $Q^{(2)}\left(\mathbf{P}_{0}(\overline{\mathbf{R}})\right)=1$. Now, from the same argument used to prove (i) there is a subsequence $\left(n^{\prime \prime}\right)$ of $\left(n^{\prime}\right)$ such that

$$
\left(\lambda_{F_{1 n^{\prime \prime}}}, \ldots, \lambda_{F_{n^{\prime \prime} n^{\prime \prime}}}, \delta_{0}, \ldots, M_{n}\left(\frac{1}{2}\right), \ldots\right) \stackrel{\ell}{\longrightarrow}\left(\delta_{0}, \ldots, 0, \ldots\right)
$$

and, therefore, the limit $Q^{(1)}$ of $Q_{n^{\prime \prime}}^{(1)}$ is concentrated on $\mathbf{P}_{0}(\overline{\mathbf{R}}) \times\left\{\delta_{0}\right\}^{\infty} \times$ $\{0\}^{\infty}$. Lemma 1 is proved.

3.1. Skorokhod's representation for a convergent subsequence of $\left(W_{n}\right)_{n}$. Let $\left(W_{n^{\prime}}\right)_{n^{\prime}}$ be a convergent subsequence of $\left(W_{n}\right)_{n}$ according to (i). Since $\mathcal{S}$ is Polish, from the Skorokhod representation theorem (cf. [2, p. 6$]$, one can determine a probability space $(\widehat{\Omega}, \widehat{\mathcal{F}}, \widehat{P})$ and random elements on it, taking values in $\mathbf{R} \times \mathbf{M}_{0}(\overline{\mathbf{R}}) \times \mathbf{P}(\overline{\mathbf{R}})^{\infty} \times \overline{\mathbf{R}}^{\infty}$ :

$$
\begin{aligned}
\widehat{W} & =\left(\widehat{\alpha}, \widehat{\lambda}_{\psi}, \widehat{\mathcal{L}}, \widehat{\mathcal{L}}_{1}, \widehat{\mathcal{L}}_{2}, \ldots, \widehat{M}\left(\frac{1}{2}\right), \widehat{M}\left(\frac{1}{3}\right), \ldots\right), \\
\widehat{W}_{n^{\prime}} & =\left(\widehat{\alpha}_{n^{\prime}}^{(r)} \widehat{\lambda}_{\psi_{n^{\prime}}^{(r)}}, \widehat{\mathcal{L}}_{n^{\prime}}, \widehat{\mathcal{L}}_{1 n^{\prime}}, \widehat{\mathcal{L}}_{2 n^{\prime}}, \ldots, \widehat{M}_{n^{\prime}}\left(\frac{1}{2}\right), \widehat{M}_{n^{\prime}}\left(\frac{1}{3}\right), \ldots\right)
\end{aligned}
$$


which have respective p.d.'s $Q$ and $Q_{n^{\prime}}$ and satisfy

$$
\lim _{n^{\prime} \rightarrow+\infty} \widehat{W}_{n^{\prime}}(\omega)=\widehat{W}(\omega) \quad \text { (in the metric of } \mathcal{S} \text { ) }
$$

for every $\omega$ in $\widehat{\Omega}$. Indeed, consistently with the previous notation, one will denote the convolution $\widehat{\mathcal{L}}_{1 n^{\prime}} * \cdots * \widehat{\mathcal{L}}_{n^{\prime} n^{\prime}}$ by $\lambda_{\widehat{\Gamma}_{n^{\prime}}}$. In view of the definition of $Q_{n}$, the following statements hold, for each $n^{\prime}, \widehat{P}$-a.s.:

$$
\begin{aligned}
& \left\{\begin{array}{l}
\left(\widehat{\mathcal{L}}_{n^{\prime}}, \widehat{\mathcal{L}}_{1 n^{\prime}}, \ldots, \widehat{\mathcal{L}}_{n^{\prime} n^{\prime}}, \delta_{0}, \delta_{0}, \ldots\right) \in \mathbf{P}_{0}(\overline{\mathbf{R}})^{\infty} \\
\hat{\mathcal{L}}_{n^{\prime}}=\lambda_{\widehat{\Gamma}_{n^{\prime}}}
\end{array}\right. \\
& \left\{\begin{array}{l}
\widehat{\alpha}_{n^{\prime}}^{(r)}=\sum_{k=1}^{n}\left\{\widehat{m}_{k n}^{(r)}+\int_{\mathbf{R}} \frac{x}{1+x^{2}} \overline{\mathcal{L}}_{k n}^{(r)}(d x)\right\} \\
\hat{\lambda}_{\psi_{n^{\prime}}^{(r)}}=\lambda_{\widehat{\psi}_{n^{\prime}}^{(r)}}
\end{array}\right.
\end{aligned}
$$

with

$$
\begin{aligned}
\widehat{m}_{k n}^{(r)} & =\int_{|x|<r} x \widehat{\mathcal{L}}_{k n}(d x), \\
\overline{\mathcal{L}}_{k n}^{(r)}(-\infty, x] & =\widehat{\mathcal{L}}_{k n}\left(-\infty, x+\widehat{m}_{k n}^{(r)}\right], \\
\widehat{\psi}_{n}^{(r)}(x) & =\sum_{k=1}^{n} \int_{(-\infty, x]} \frac{y^{2}}{1+y^{2}} \overline{\mathcal{L}}_{k n}^{(r)}(d y), \\
\widehat{M}_{n^{\prime}}\left(\frac{1}{k}\right) & =\max _{1 \leqslant i \leqslant n^{\prime}} \widehat{\mathcal{L}}_{i n^{\prime}}\left(\left[-\frac{1}{k}, \frac{1}{k}\right]^{c}\right), \quad k=2,3, \ldots,
\end{aligned}
$$

and, from (2.1):

$$
\begin{aligned}
& \widehat{M}_{n^{\prime}}\left(\frac{1}{k}\right) \rightarrow 0, \quad n^{\prime} \rightarrow \infty ; \quad k=2,3, \ldots, \\
& \widehat{\mathcal{L}}_{i n^{\prime}} \Rightarrow \widehat{\mathcal{L}}_{i}=\delta_{0}, \quad i=1,2, \ldots .
\end{aligned}
$$

Moreover from (3.5):

$$
\max _{1 \leqslant i \leqslant n^{\prime}} \widehat{\mathcal{L}}_{i n^{\prime}}\left([-\varepsilon, \varepsilon]^{c}\right) \longrightarrow 0, \quad n^{\prime} \rightarrow \infty, \quad \varepsilon>0
$$

holds $\widehat{P}$-a.s. In other words, p.d.'s $\widehat{\mathcal{L}}_{i n^{\prime}}$ are u.a.n., $\widehat{P}$-a.s. Now, in view of (3.2), (3.3) and (3.7) the conditions of the general version of the central limit theorem, for independent summands, hold $\widehat{P}$-a.s.; cf. [16, p. 321, A $\left.2^{\circ}\right]$. Hence, $\widehat{\mathcal{L}} \in \mathbf{P}_{0}(\overline{\mathbf{R}}) \widehat{P}$-a.s. and

$$
\int_{\mathbf{R}} e^{i t x} \widehat{\mathcal{L}}(d x)=h(t ; \widehat{\alpha}, \widehat{\psi}), \quad t \in \mathbf{R},
$$


with

$$
h(t ; \widehat{\alpha}, \widehat{\psi})=\exp \left\{i t \hat{\alpha}+\int_{\mathbf{R}}\left(e^{i t u}-1-\frac{i t u}{1+u^{2}}\right) \frac{1+u^{2}}{u^{2}} \lambda_{\widehat{\psi}}(d u)\right\} .
$$

It should de noted that $Q$ is concentrated on $\mathbf{R} \times \mathbf{M}_{0}(\overline{\mathbf{R}}) \times \mathbf{P}_{0}(\overline{\mathbf{R}}) \times$ $\left\{\delta_{0}\right\}^{\infty} \times\{0\}^{\infty}$.

3.2. Skorokhod's representation for a convergent subsequence of $\left(W_{n}^{(1)}\right)_{n}$. If $\left(W_{n^{\prime}}^{(1)}\right)_{n^{\prime}}$ is a convergent subsequence of $\left(W_{n}^{(1)}\right)_{n}$ according to (ii), from the Skorokhod representation theorem, there are random elements taking values in $\mathbf{P}_{0}(\overline{\mathbf{R}})^{\infty} \times \overline{\mathbf{R}}^{\infty}$ :

$$
\begin{aligned}
& \widehat{W}^{(1)}=\left(\widehat{\mathcal{L}}, \widehat{\mathcal{L}}_{1}, \widehat{\mathcal{L}}_{2}, \ldots, \widehat{M}\left(\frac{1}{2}\right), \widehat{M}\left(\frac{1}{3}\right), \ldots\right), \\
& \widehat{W}_{n^{\prime}}^{(1)}=\left(\widehat{\mathcal{L}}_{n^{\prime}}, \widehat{\mathcal{L}}_{1 n^{\prime}}, \widehat{\mathcal{L}}_{2 n^{\prime}}, \ldots, \widehat{M}_{n^{\prime}}\left(\frac{1}{2}\right), \widehat{M}_{n^{\prime}}\left(\frac{1}{3}\right), \ldots\right),
\end{aligned}
$$

which have respective p.d.'s $Q^{(1)}$ and $Q_{n^{\prime}}^{(1)}$ and satisfy (3.3) and (3.5)-(3.7) and

$$
\lim _{n^{\prime} \rightarrow+\infty} \widehat{W}_{n^{\prime}}^{(1)}(\omega)=\widehat{W}^{(1)}(\omega) \quad \text { (in the metric of } \mathcal{S}^{(1)} \text { ) }
$$

for every $\omega$ in $\widehat{\Omega}$. Consequently, from the general version of the central limit theorem for independent summands (cf. [16, p. 321]), there exist random elements $\hat{\alpha}$ taking value in $\mathbf{R}$ and $\lambda_{\widehat{\psi}}$ taking value in $\mathbf{M}_{0}(\overline{\mathbf{R}})$ such that

$$
\int_{\mathbf{R}} e^{i t x} \widehat{\mathcal{L}}(d x)=h(t ; \widehat{\alpha}, \widehat{\psi}), \quad t \in \mathbf{R}
$$

with

$$
h(t ; \widehat{\alpha}, \widehat{\psi})=\exp \left\{i t \widehat{\alpha}+\int_{\mathbf{R}}\left(e^{i t u}-1-\frac{i t u}{1+u^{2}}\right) \frac{1+u^{2}}{u^{2}} \lambda_{\widehat{\psi}}(d u)\right\}
$$

and

$$
\left\{\begin{array}{l}
\widehat{\alpha}_{n^{\prime}}^{(r)} \longrightarrow \widehat{\alpha}, \\
\lambda_{\widehat{\psi}_{n^{\prime}}^{(r)}} \longrightarrow \lambda_{\widehat{\psi}} \quad \text { completely, } \quad n^{\prime} \rightarrow+\infty,
\end{array}\right.
$$

$\widehat{P}$-a.s. for every $r>0$.

\section{A central limit theorem for sums of partially exchangeable r.v.'s}

The present section provides a solution, under conditions $\left(\mathrm{H}_{1}\right)-\left(\mathrm{H}_{2}\right)$, for the problem formulated in Section 2. The proof of the main result is based on the Skorokhod representations in Subsections 3.1 and 3.2. 
Theorem 1. Assume that $\left(\mathrm{H}_{1}\right)-\left(\mathrm{H}_{2}\right)$ hold. Then

$\left.1^{\circ}\right)$ If $\left(\left(S_{1 n}, S_{2 n}, \ldots\right)\right)_{n}$ converges in law, then there is a unique probability measure $\nu$ on $\left(\mathbf{R} \times \mathbf{M}_{0}(\overline{\mathbf{R}}), \mathcal{B}\left(\mathbf{R} \times \mathbf{M}_{0}(\overline{\mathbf{R}})\right)\right)$ such that the limiting law of $\left(\left(S_{1 n}, \ldots, S_{m n}\right)\right)_{n}$ has the following Fourier-Stieltjes transform

$$
\begin{gathered}
\varphi_{m}\left(t_{1}, \ldots, t_{m}\right) \\
=\int_{\mathbf{R} \times \mathbf{M}_{0}(\overline{\mathbf{R}})} \prod_{k=1}^{m} \exp \left\{i \alpha t_{k}+\int_{\mathbf{R}}\left(e^{i t_{k} x}-1-\frac{i t_{k} x}{1+x^{2}}\right) \frac{1+x^{2}}{x^{2}} \rho(d x)\right\} \\
\times \nu(d \alpha d \rho),
\end{gathered}
$$

$\left(t_{1}, \ldots, t_{m}\right) \in \mathbf{R}^{m}, m=1,2, \ldots$.

$\left.2^{\circ}\right)\left(\left(S_{1 n}, S_{2 n}, \ldots\right)\right)_{n}$ converges in law to a random sequence whose p.d. is characterized by (4.1) if, and only if, $\nu_{n}^{(r)}$ defined by (2.5) satisfies

$$
\nu_{n}^{(r)} \Rightarrow \nu, \quad n \rightarrow \infty,
$$

for any fixed finite $r>0$.

P r o of. If $\left(\left(S_{1 n}, S_{2 n}, \ldots\right)\right)_{n}$ converges in law, then, according to de Finetti's representation theorem, the limiting c.f. of $\left(S_{1 n}, \ldots, S_{m n}\right)$ is given by

$$
\lim _{n \rightarrow \infty} E\left(\exp \left\{i \sum_{k=1}^{m} t_{k} S_{k n}\right\}\right)=\lim _{n \rightarrow \infty} E\left(\prod_{k=1}^{m} \int e^{i t_{k} x} \lambda_{\Gamma_{n}}(d x)\right) .
$$

Furthermore, from Lemma 1 (ii), every subsequence of $(n)$ includes a subsequence $\left(n^{\prime}\right)$ such that

$$
\lim _{n \rightarrow \infty} E\left(\prod_{k=1}^{m} \int e^{i t_{k} x} \lambda_{\Gamma_{n}}(d x)\right)=\lim _{n^{\prime} \rightarrow \infty} \hat{E}\left(\prod_{k=1}^{m} \int e^{i t_{k} x} \lambda_{\widehat{\Gamma}_{n}^{\prime}}(d x)\right)
$$

(from the Skorokhod representation in Subsection 3.2)

$$
=\widehat{E}\left(\prod_{k=1}^{m} h\left(t_{k} ; \widehat{\alpha}, \widehat{\psi}\right)\right)
$$

(from (3.8) and the Lebesgue dominated convergence theorem).

Then, if $\nu$ represents the p.d. of $\left(\widehat{\alpha}, \lambda_{\widehat{\psi}}\right)$ on $\left(\mathbf{R} \times \mathbf{M}_{0}(\overline{\mathbf{R}}), \mathcal{B}\left(\mathbf{R} \times \mathbf{M}_{0}(\overline{\mathbf{R}})\right)\right)$, one obtains

$$
\widehat{E}\left(\prod_{k=1}^{m} h\left(t_{k} ; \widehat{\alpha}, \widehat{\psi}\right)\right)=\int_{\mathbf{R} \times \mathbf{M}_{0}} \prod_{k=1}^{m} h\left(t_{k} ; \alpha, \rho\right) \nu(d \alpha d \rho)
$$

for every $\left(t_{1}, \ldots, t_{m}\right)$ in $\mathbf{R}^{m}$ and every $m$. Hence, there is a random sequence whose p.d. is determined by the right-hand side of (4.4). The elements of 
this sequence are exchangeable and, consequently, uniqueness of $\nu$ follows from de Finetti's representation theorem, and this completes the proof of the first part of the theorem.

To prove the second part, firstly assume that $\left(S_{1 n}, \ldots, S_{m n}\right)_{n}$ converges in law. Then from Subsection 3.2, $\widehat{\mathcal{L}}$ belongs to $\mathbf{P}_{0}(\overline{\mathbf{R}})$ and, thanks to Subsection 3.1, $\widehat{\mathcal{L}}$ is the weak limit of $\lambda_{\widehat{\Gamma}_{n},} \widehat{P}$-a.s. Consequently from the central limit theorem (cf. [16, A $1^{\circ}$, p. 321]) the Fourier-Stieltjes transform of $\widehat{\mathcal{L}}$ is given by (3.9), $\widehat{P}$-a.s. and the p.d. $\nu$ of $\left(\widehat{\alpha}, \lambda_{\widehat{\psi}}\right)$ is the weak limit of $\nu_{n^{\prime}}^{(r)}$. At this stage, (4.2) follows from the uniqueness of $\nu$ stated in the first part of the theorem. To prove the converse, it suffices to consider the c.f. $\varphi_{m}^{(n)}$ of $\left(S_{1 n}, \ldots, S_{m n}\right)$ and to prove that every pointwise convergent subsequence of $\left(\varphi_{m}^{(n)}\right)_{n}$ converges to the right-hand side of (4.1), for $m=1,2, \ldots$ If $(4.2)$ holds, the Subsection 3.1 yields

$$
\prod_{k=1}^{m} \int_{\mathbf{R}} e^{i t_{k} x} \lambda_{\widehat{\Gamma}_{n^{\prime}}}(d x) \longrightarrow \prod_{k=1}^{m} h\left(t_{k} ; \widehat{\alpha}, \widehat{\psi}\right), \quad n^{\prime} \rightarrow \infty,
$$

for every $\left(t_{1}, \ldots, t_{m}\right)$ in $\mathbf{R}^{m}, \widehat{P}$-a.s., and in view of (3.2) and (4.2) the p.d. of $\left(\widehat{\alpha}, \lambda_{\hat{\psi}}\right)$ is $\nu$. Hence, from the Lebesgue dominated convergence theorem,

$$
\lim _{n^{\prime}} \varphi_{m}^{\left(n^{\prime}\right)}\left(t_{1}, \ldots, t_{m}\right)=\int_{\mathbf{R} \times \mathbf{M}_{0}(\overline{\mathbf{R}})} \prod_{k=1}^{m} h\left(t_{k} ; \alpha, \rho\right) \nu(d \alpha d \rho)
$$

and this completes the proof.

It may happen that, under $\left(\mathrm{H}_{1}\right)-\left(\mathrm{H}_{2}\right)$, the sequence $\left(\left(S_{1 n}, S_{2 n}, \ldots\right)\right)_{n}$ does not converge in law but the sequence $\left(\left(S_{1 n}-c_{n}, S_{2 n}-c_{n}, \ldots\right)\right)_{n}$ does for suitable constants $c_{n}, n=1,2, \ldots$. The following extension of Theorem 1 is immediate.

Theorem 2. Suppose $\left(\mathrm{H}_{1}\right),\left(\mathrm{H}_{2}\right)$ hold, and let $\left(c_{n}\right)_{n}$ be a sequence of real numbers. Then

$\left.1^{\circ}\right)$ If $\left(\left(S_{1 n}-c_{n}, S_{2 n}-c_{n}, \ldots\right)\right)_{n}$ converges in law, then there is a unique probability measure $\nu$ on $\left(\mathbf{R} \times \mathbf{M}_{0}(\overline{\mathbf{R}}), \mathcal{B}\left(\mathbf{R} \times \mathbf{M}_{0}(\overline{\mathbf{R}})\right)\right)$ such that (4.1) is the Fourier-Stieltjes transform of the limiting law of $\left(\left(S_{1 n}-c_{n}, \ldots, S_{m n}-c_{n}\right)\right)_{n}$.

$\left.2^{\circ}\right)\left(\left(S_{1 n}-c_{n}, S_{2 n}-c_{n}, \ldots\right)\right)_{n}$ converges in law to a random sequence whose p.d. is characterized by (4.1) if, and only if,

$$
\widetilde{\nu}_{n}^{(r)} \Rightarrow \nu, \quad n \rightarrow \infty
$$

with $\widetilde{\nu}_{n}^{(r)}(A \times B)=P\left(\alpha_{n}^{(r)}-c_{n} \in A, \lambda_{\psi_{n}^{(r)}} \in B\right)$ for every $A \times B \in \mathcal{B}(\mathbf{R} \times$ $\mathbf{M}_{0}(\overline{\mathbf{R}})$ ) and for any fixed $r>0$.

In Section 6 we provide a sharper characterization of the limiting law in point $1^{\circ}$ of Theorem 2, when $\xi_{i k}^{(n)}=X_{i k} / a_{n}, a_{n}>0$ for every $n, a_{n} \rightarrow+\infty$ and $X_{i k}(i, k=1,2, \ldots)$ are exchangeable r.v.'s. 


\section{Convergence to mixtures of Gaussian, Poisson, degenerate p.d.'s}

This section deals with central limit criteria for three noteworthy limit types: mixtures of Gaussian, Poisson, degenerate p.d.'s. After choosing $r>0$, define $\pi_{n}^{(r)}$ to be the p.d. of $\left(m_{n}(\omega, r), \sigma_{n}^{2}(\omega, r)\right)$ with

$$
\begin{aligned}
m_{n}(\omega, r) & =\sum_{k=1}^{n} \int_{|x|<r} x d F_{k n}(x, \omega) \\
\sigma_{n}^{2}(\omega, r) & =\sum_{k=1}^{n}\left\{\int_{|x|<r} x^{2} d F_{k n}(x, \omega)-\left(\int_{|x|<r} x d F_{k n}(x, \omega)\right)^{2}\right\} .
\end{aligned}
$$

Moreover, for every $\varepsilon>0$, let $q_{n}^{(\varepsilon)}$ be the p.d. of $\sum_{k=1}^{n} \int_{|x| \geqslant \varepsilon} d F_{k n}(x, \omega)$.

Theorem 3. Suppose that $\left(\mathrm{H}_{1}\right)$ holds. Then $\left(\mathrm{H}_{2}\right)$ holds and there is a p.d. $\pi$ on the Borel sets of $\mathbf{R} \times \mathbf{R}_{+}\left(\right.$where $\left.\mathbf{R}_{+}=[0,+\infty)\right)$ such that

$$
\begin{gathered}
\lim _{n \rightarrow \infty} E\left(\exp \left\{i \sum_{k=1}^{m} t_{k} S_{k n}\right\}\right)=\int_{\mathbf{R} \times \mathbf{R}_{+}} \prod_{k=1}^{m} \exp \left\{i t_{k} a-\frac{1}{2} t_{k}^{2} \sigma^{2}\right\} \pi\left(d a d \sigma^{2}\right) \\
\left.\left(t_{1}, \ldots, t_{m}\right) \in \mathbf{R}^{m}\right), \quad m=1,2, \ldots
\end{gathered}
$$

if, and only if,

$$
\left\{\begin{array}{l}
\pi_{n}^{(r)} \Rightarrow \pi \\
q_{n}^{(\varepsilon)} \Rightarrow \delta_{0}
\end{array}\right.
$$

holds for some $r>0$ and for every $\varepsilon>0$, as $n \rightarrow \infty$.

$\mathrm{P}$ r o o f. Necessity. In view of (5.1), point $\left.1^{\circ}\right)$ of Theorem 1 and the uniqueness of $\nu$, one obtains that $\nu$ coincides with a p.d. $\pi$ supported by $\mathbf{R} \times \mathbf{D}_{0}$, where $\mathbf{D}_{0}$ is the set of all masses concentrated at 0 . Hence, from the Skorokhod representation in Subsection 3.2, $\widehat{\mathcal{L}}_{n^{\prime}}$ converges weakly to the Gaussian law with mean $\widehat{\alpha}$ and variance $\widehat{\sigma}^{2}, \widehat{P}$-a.s. Moreover, the Gaussian and degenerate convergence criteria for independent summands $[16$, p. 328329] yields

$$
\begin{gathered}
\widehat{\sigma}_{n^{\prime}}^{2}(\omega, r) \longrightarrow \widehat{\sigma}^{2}(\omega), \quad \widehat{m}_{n^{\prime}}(\omega, r) \longrightarrow \widehat{\alpha}(\omega), \\
\sum_{k=1}^{n^{\prime}} \int_{|x| \geqslant \varepsilon} \widehat{\mathcal{L}}_{k n^{\prime}}(d x, \omega) \longrightarrow 0,
\end{gathered}
$$

$\widehat{P}_{\text {-a.s., }}$ where $\widehat{m}_{n^{\prime}}, \widehat{\sigma}_{n^{\prime}}^{2}$ are the same as $m_{n^{\prime}}, \sigma_{n^{\prime}}^{2}$, respectively, with $\widehat{\mathcal{L}}_{k n^{\prime}}$ in the place of ${ }^{\prime} \lambda_{F_{k n}}$. In fact, each subsequence of $(n)$ includes a subsequence, $\left(n^{\prime}\right)$, for which (5.3)-(5.4) hold and this - via the Prokhorov theorem implies (5.2).

Sufficiency. To prove the converse, one can observe that $\left(\mathrm{H}_{2}\right)$ easily follows from $q_{n}^{(\varepsilon)} \Rightarrow \delta_{0}$. Moreover, if

$$
Z_{n}=\left(W_{n}^{(1)}, U_{n}\right), \quad n=1,2, \ldots,
$$


where $W_{n}^{(1)}$ is defined at the beginning of Section 3 and

$$
U_{n}=\left(m_{n}(\cdot, r), \sigma_{n}^{2}(\cdot, r), \sum_{k=1}^{n} \int_{|x| \geqslant 1 / 2} d F_{k n}, \sum_{k=1}^{n} \int_{|x| \geqslant 1 / 3} d F_{k n}, \ldots\right),
$$

then the sequence of the p.d.'s of $Z_{1}, Z_{2}, \ldots$ is tight. Hence, every subsequence of $(n)$ admits a subsequence $\left(n^{\prime}\right)$ for which a representation $a$ la Skorokhod holds, and this representation is analogous to the one exhibited in Subsection 3.1. In particular, one can state the existence of a random vector $\left(\widehat{\alpha}, \hat{\sigma}^{2}\right)$, taking values in $\mathbf{R} \times \mathbf{R}_{+}$whose p.d. is $\pi$. Moreover (5.3)(5.4) and (3.7) hold and, consequently, from the above mentioned Gaussian convergence criterion:

$$
\prod_{k=1}^{m} \int_{\mathbf{R}} \exp \left\{i t_{k} x\right\} \lambda_{\widehat{\Gamma}_{n^{\prime}}}(d x) \rightarrow \prod_{k=1}^{m} \exp \left\{i t_{k} \widehat{\alpha}-\frac{t_{k}^{2} \widehat{\sigma}^{2}}{2}\right\}, \quad n^{\prime} \rightarrow \infty,
$$

obtains on $\widehat{\Omega}_{1}$, with $\widehat{P}\left(\widehat{\Omega}_{1}\right)=1$, for every $\left(t_{1}, \ldots, t_{m}\right)$ in $\mathbf{R}^{m}$ and $m=1,2, \ldots$ Then; an application of the dominated convergence theorem leads to the formula

$$
\lim _{n^{\prime}} E\left(\exp \left\{i \sum_{k=1}^{m} t_{k} S_{k n^{\prime}}\right\}\right)=\int_{\mathbf{R} \times \mathbf{R}_{+}} \prod_{k=1}^{m} \exp \left\{i t_{k} a-\frac{t_{k}^{2}}{2} \sigma^{2}\right\} \pi\left(d a d \sigma^{2}\right)
$$

and (5.1) follows from the Prokhorov theorem. Theorem 3 is proved.

The proof of the following extended version of Theorem 3 is immediate.

Theorem 4. Suppose $\left(\mathrm{H}_{1}\right)$ holds. Then $\left(\mathrm{H}_{2}\right)$ holds and there exist a sequence of real numbers $\left(c_{n}\right)_{n}$ and a p.d. $\tilde{\pi}$ on the Borel sets of $\mathbf{R} \times \mathbf{R}_{+}$ such that

$$
\begin{aligned}
\lim _{n \rightarrow \infty} & E\left(\exp \left\{i \sum_{k=1}^{m} t_{k}\left(S_{k n}-c_{n}\right)\right\}\right) \\
= & \int_{\mathbf{R} \times \mathbf{R}_{+}} \prod_{k=1}^{m} \exp \left\{i t_{k} a-\frac{1}{2} t_{k}^{2} \sigma^{2}\right\} \tilde{\pi}\left(d a d \sigma^{2}\right), \\
& \left(t_{1}, \ldots, t_{m}\right) \in \mathbf{R}^{m}, \quad m=1,2, \ldots,
\end{aligned}
$$

if, and only if, for every $\varepsilon>0$ and some $r>0$ :

$$
\left\{\begin{array}{l}
\tilde{\pi}_{n}^{(r)} \Rightarrow \tilde{\pi}, \quad n \rightarrow \infty \\
q_{n}^{(\varepsilon)} \Rightarrow \delta_{0},
\end{array}\right.
$$

where $\widetilde{\pi}_{n}^{(r)}$ is the p.d. of $\left(m_{n}(\cdot, r)-c_{n}, \sigma_{n}^{2}(\cdot, r)\right)$.

By an analogous line of reasoning, one can state a Poisson convergence criterion. 
Theorem 5. Under $\left(\mathrm{H}_{1}\right)$ and $\left(\mathrm{H}_{2}\right)$, there is a probability measure $\chi$ on $\mathcal{B}\left(\mathbf{R}_{+}\right)$such that

$$
\lim _{n \rightarrow \infty} E\left(\exp \left\{i \sum_{k=1}^{m} t_{k} S_{k n}\right\}\right)=\int_{\mathbf{R}_{+}} \prod_{k=1}^{m} \exp \left\{\lambda\left(e^{i t_{k}}-1\right)\right\} \chi(d \lambda)
$$

holds for every $\left(t_{1}, \ldots, t_{m}\right)$ in $\mathbf{R}^{m}(m=1,2 \ldots)$ if, and only if, for every $\varepsilon$ in $(0,1)$ and some $r$ in $(0,1)$ :

$$
\sum_{k=1}^{n} \int_{|x| \geqslant \varepsilon,|x-1| \geqslant \varepsilon} d F_{k n}(x, \omega) \stackrel{P}{\longrightarrow} 0, \quad \sigma_{n}^{2}(\omega, r) \stackrel{P}{\longrightarrow} 0, \quad m_{n}(\omega, r) \stackrel{P}{\longrightarrow} 0
$$

and

$$
\chi_{n}^{(\varepsilon)} \Rightarrow \chi,
$$

$\chi_{n}^{(\varepsilon)}$ being the p.d. of $\sum_{k=1}^{n} \int_{|x-1|<\varepsilon} d F_{k n}(x, \omega), n=1,2, \ldots$

Since the degenerate law can be considered as a degenerate Gaussian law, the following proposition immediately follows from Theorem 4.

Corollary 1. If $\left(\mathrm{H}_{1}\right)$ is valid, then $\left(\mathrm{H}_{2}\right)$ holds and there exist a sequence of real numbers $\left(c_{n}\right)_{n}$ and a probability measure $\kappa$ on $\mathcal{B}(\mathbf{R})$ such that

$$
\begin{gathered}
\lim _{n \rightarrow \infty} E\left(\exp \left\{i \sum_{k=1}^{m} t_{k}\left(S_{k n}-c_{n}\right)\right\}\right) \\
\left(t_{1}, \ldots, t_{m}\right) \in \mathbf{R}^{m}, \quad m=1,2, \ldots,
\end{gathered}
$$

if, and only if, for every $\varepsilon>0$ and for some $r>0$,

$$
\sum_{k=1}^{n} \int_{|x| \geqslant \varepsilon} d F_{k n}(x, \omega) \stackrel{P}{\longrightarrow} 0, \quad \sigma_{n}^{2}(\omega, r) \stackrel{P}{\longrightarrow} 0
$$

and

$$
\kappa_{n}^{(r)} \Rightarrow \kappa, \quad n \rightarrow \infty,
$$

where $\kappa_{n}^{(r)}$ denotes the p.d. of $\left(m_{n}(\omega, \cdot)-c_{n}\right)$.

\section{Exchangeable summands. A few remarks} when:

The present section includes some comments on Theorem 2, point $1^{\circ}$ ), $\xi_{i j}^{(n)}=\frac{X_{i j}}{a_{n}}$, where $\left\{X_{i j} ; i, j \geqslant 1\right\}$ is an array of exchangeable r.v.'s and $\left(a_{n}\right)_{n}$ is a divergent sequence of strictly positive numbers;

$c_{n}=\frac{b_{n}}{a_{n}},\left(b_{n}\right)_{n}$ being a sequence of real numbers; 


$$
S_{1 n}-c_{n}=\frac{\left(\sum_{j=1}^{n} X_{1 j}-b_{n}\right)}{a_{n}} \text { converges in law to a }
$$

nondegenerate r.v.

Notice that here one does not require convergence of $\left(S_{1 n}-c_{n}, S_{2 n}-\right.$ $\left.c_{n}, \ldots\right)$. Under the above assumptions, one can prove the following result which will be used to provide a new proof for Theorem 2 in [13]; see Subsection 7.4.

Theorem 6. If (6.1)-(6.3) hold, then:

$\left.1^{\circ}\right)$ Each subsequence of $(n)$ includes a subsequence $\left(n^{\prime}\right)$ such that $\left(a_{r n^{\prime}} /\right.$ $\left.a_{n^{\prime}}\right)_{n^{\prime}}$ converges to a strictly positive limit, $\alpha^{(r)}$, and $\left(\left(b_{r n^{\prime}}-r b_{n^{\prime}}\right) / a_{n^{\prime}}\right)_{n^{\prime}}$ converges to $\beta^{(r)}$, for any positive integer $r$.

$\left.2^{\circ}\right)$ There exists a probability measure $P^{*}$ on $\left(\mathbf{R}^{\infty}, \mathcal{B}\left(\mathbf{R}^{\infty}\right)\right)$ such that the limiting c.f. $\phi$ of $\left(S_{1 n}-c_{n}\right)_{n}$ admits the representation

$$
\phi(t)=\int_{\mathbf{R}^{\infty}} g(t, \omega) P^{*}(d \omega), \quad t \in \mathbf{R},
$$

where $g(\cdot, \omega)$ is a c.f. for every $\omega$ in $\mathbf{R}^{\infty}$ and

$$
P^{*}\left(g(t, \omega)=g\left(\frac{t}{\alpha^{(r)}}, \omega\right)^{r} e^{i t \beta^{(r)} / \alpha^{(r)}} \quad \text { for all } t \text { in } \mathbf{R}\right)=1 .
$$

P r o of. We start by observing that

$$
S_{j n}-c_{n} \stackrel{\ell}{=} S_{1 n}-c_{n}, \quad j \geqslant 2,
$$

where $\stackrel{\ell}{=}$ denotes equality in law. Then, the sequence of p.d.'s of $V_{n}^{(r)}:=$ $\left(S_{1 n}-c_{n}, \ldots, S_{r n}-c_{n}\right), n=1,2, \ldots$, is tight for every $r$. Hence, by resorting to the diagonal method, one can prove that each subsequence of $\left(V_{n}^{(r)}\right)_{n}$ includes a subsequence $\left(V_{n^{\prime}}^{(r)}\right)_{n^{\prime}}$ such that

$$
V_{n^{\prime}}^{(r)} \stackrel{\ell}{\longrightarrow}\left(X_{1}, X_{2}, \ldots\right) \text {. }
$$

From (6.5),

$$
W_{n^{\prime}}^{(r)}=\sum_{k=1}^{r}\left(S_{k n^{\prime}}-c_{n^{\prime}}\right) \stackrel{\ell}{\longrightarrow} X_{1}+\cdots+X_{r}, \quad r=1,2, \ldots,
$$

and, since

$$
W_{n}^{(r)} \stackrel{\ell}{=} \frac{a_{r n}}{a_{n}}\left(S_{1 r n}-c_{r n}\right)+\frac{b_{r n}-r b_{n}}{a_{n}}, \quad n=1,2, \ldots,
$$

the convergence of types theorem implies that there exist $\alpha^{(r)}>0$ and $\beta^{(r)}$ such that

$$
\frac{a_{r n^{\prime}}}{a_{n^{\prime}}} \longrightarrow \alpha^{(r)}, \quad \frac{b_{r n^{\prime}}-r b_{n^{\prime}}}{a_{n^{\prime}}} \longrightarrow \beta^{(r)}, \quad n^{\prime} \rightarrow \infty
$$


Now, from (6.6)-(6.8) and (6.3),

$$
X \stackrel{\ell}{=} \frac{X_{1}+\cdots+X_{r}-\beta^{(r)}}{\alpha^{(r)}}, \quad r=1,2, \ldots,
$$

where $X$ is the limit (in law) of $\left(S_{1 n}-c_{n}\right)_{n}$. If $P^{*}$ denotes the p.d. of $\left(X_{n}\right)_{n}$, then, since the $X_{n}$ 's are exchangeable, one obtains the representation

$$
E\left(\exp \left(i \sum_{k=1}^{r} t_{k} X_{k}\right)\right)=\int_{\mathbf{R}^{\infty}} \prod_{k=1}^{r} g\left(t_{k}, \omega\right) P^{*}(d \omega)
$$

For each $\left(n^{\prime}\right)$ such that $(6.5)$ holds, this representation is unique. Clearly, it generally depends on $\left(n^{\prime}\right)$. Then, since $X \stackrel{\ell}{=} X_{n},(6.4)$ follows from (6.9). Theorem 6 is proved.

We now investigate the admissible values of $\alpha^{(r)}$. In fact it follows from $(6.4)$ that $g(\cdot, \omega)$ turns out to be, $P^{*}$-a.s., the c.f. of a stable law and, therefore,

$$
g(t, \omega)=\exp \left\{i t \gamma(\omega)-c(\omega)|t|^{\eta(\omega)}\left[1+i \beta(\omega) \frac{t}{|t|} w(t, \eta(\omega))\right]\right\}
$$

for all $t \in \mathbf{R}$ where $\eta, \beta, c$ are r.v.'s such that $0<\eta(\omega) \leqslant 2,|\beta(\omega)| \leqslant 1$, $c(\omega) \geqslant 0$,

$$
w(t, \eta(\omega))=\left\{\begin{array}{lll}
\operatorname{tang} \frac{\pi \eta(\omega)}{2} & \text { if } & \eta(\omega) \neq 1 \\
\frac{2}{\pi} \log |t| & \text { if } & \eta(\omega)=1
\end{array}\right.
$$

Hence, by (6.4), the following system of equations holds $P^{*}$-a.s., for every positive integer $r$

$$
\left\{\begin{array}{l}
\gamma(\omega)\left[1-\frac{r}{\alpha^{(r)}}\right]=\frac{\beta^{(r)}}{\alpha^{(r)}} \\
c(\omega)\left[1-\frac{r}{\left(\alpha^{(r)}\right)^{\eta(\omega)}}\right]=0 \\
c(\omega) \beta(\omega)\left[w(t, \eta(\omega))-\frac{r}{\left(\alpha^{(r)}\right)^{\eta(\omega)}} w\left(\frac{t}{\alpha^{(r)}}, \eta(\omega)\right)\right]=0
\end{array}\right.
$$

Then, if

$$
\alpha^{(r)}=r \quad \text { for every positive integer } r
$$

either

$$
c(\omega)=0
$$

or

$$
\left\{\begin{array}{l}
c(\omega) \neq 0 \\
\eta(\omega)=1 \\
\beta(\omega)=0
\end{array}\right.
$$

5 Теория верохтностей и ее применения, № 2 
holds. On the other hand, if (6.13) does not hold, then either

$$
\left\{\begin{array}{l}
c(\omega)=0 \\
\gamma(\omega)=\gamma
\end{array}\right.
$$

or

$$
\left\{\begin{array}{l}
c(\omega) \neq 0 \\
\gamma(\omega)=\gamma \\
\eta(\omega)=\eta \neq 1 \\
\alpha^{(r)}=r^{1 / \eta}
\end{array}\right.
$$

obtains. Hence, if (6.13) does not hold, since the limiting p.d. of $\left(S_{1 n}-c_{n}\right)_{n}$ is nondegenerate, then the probability that $(6.17)$ holds is strictly positive. This proves

Theorem 7. If (6.1)-(6.3) hold, then either (6.13) or

$$
\alpha^{(r)}=r^{1 / \eta} \quad \text { for some } \quad \eta \neq 1 \text { and for every } \quad r
$$

holds.

For suitable choices of the limiting c.f. $\phi$, one can show that (6.1)-(6.3) imply convergence of $\left(\left(S_{1 n}-c_{n}, S_{2 n}-c_{n}, \ldots\right)\right)_{n}$. In particular, this comes true when (6.3) is replaced by

$S_{1 n}-c_{n}$ converges in law to a standard Gaussian r.v., say $Z$. $\quad\left(6.3^{\prime}\right)$

Theorem 8. If (6.1), (6.2) and (6.3') hold, then $\left(\left(S_{1 n}-c_{n}, S_{2 n}-\right.\right.$ $\left.\left.c_{n}, \ldots\right)\right)_{n}$ converges in law.

$\mathrm{Pr}$ o of. In view of the arguments used to obtain Theorem 7 , the limiting c.f. $\phi_{Z}$ of $Z$ admits one of the following two representations:

$$
\phi_{Z}(t)=\int_{\mathbf{R}^{\infty}} \exp \{i \gamma(\omega) t-c(\omega)|t|\} P^{*}(d \omega)
$$

when (6.13) holds;

$$
\phi_{Z}(t)=\int_{\mathbf{R}^{\infty}} \exp \left\{i \gamma t-c(\omega)|t|^{\eta}\left[1+i \beta(\omega) \frac{t}{|t|} \operatorname{tang} \frac{\pi \eta}{2}\right]\right\} P^{*}(d \omega)
$$

when (6.18) occurs. Since $\phi_{Z}(t)=\exp \left(-t^{2} / 2\right)$ is infinitely differentiable, then

$$
\begin{array}{lll}
c(\omega)=0 & \text { in }(6.19) \\
\eta=2, \gamma=0, c(\omega)=\frac{1}{2} & \text { in } \quad(6.20)
\end{array} \quad P^{*} \text {-a.s. }
$$

From (6.8), (6.13), Theorem 7 and (6.21), each convergent subsequence of $\left(a_{r n} / a_{n}\right)_{n}$ converges either to $\sqrt{r}$ or to $r$. Hence, given any positive integer $r$, define

$$
\begin{aligned}
& N_{1}(r)=\left\{n \in \mathbf{N}:\left|\frac{a_{r n}}{a_{n}}-\sqrt{r}\right|<\left|\frac{a_{r n}}{a_{n}}-r\right|\right\}, \\
& N_{2}(r)=\mathbf{N}-N_{1}(r) .
\end{aligned}
$$


If $N_{1}(r)$ is infinite $\left(N_{2}(r)\right.$ infinite), then

$$
\lim _{\substack{n^{\prime} \rightarrow+\infty \\ n^{\prime} \in N_{1}(r)}} \frac{a_{r n^{\prime}}}{a_{n^{\prime}}}=\sqrt{r}\left[\lim _{\substack{n^{\prime \prime} \\ n^{\prime \prime} \in N_{2}(r)}} \frac{a_{r n^{\prime \prime}}^{+\infty}}{a_{n^{\prime \prime}}}=r\right]
$$

for some suitable subsequence $\left(n^{\prime}\right)\left(\left(n^{\prime \prime}\right)\right)$. Moreover, if $N_{1}(r)$ and $N_{2}(r)$ are both infinite then $\left(n^{\prime}\right)$ and $\left(n^{\prime \prime}\right)$ can be chosen in such a way that $n_{k}^{\prime \prime}=n_{k}^{\prime}+1$. This implies

$$
\begin{aligned}
\frac{S_{1 n_{k}^{\prime \prime} r}-b_{n_{k}^{\prime \prime} r}}{a_{n_{k}^{\prime \prime} r}}= & \frac{S_{1 n_{k}^{\prime} r}-b_{n_{k}^{\prime} r}}{a_{n_{k}^{\prime} r}} \frac{a_{n_{k}^{\prime} r}}{a_{n_{k}^{\prime \prime} r}}+\frac{b_{n_{k}^{\prime} r}-b_{n_{k}^{\prime \prime} r}}{a_{n_{k}^{\prime \prime} r}} \\
& +\frac{X_{1 n_{k}^{\prime} r+1}+\cdots+X_{1\left(n_{k}^{\prime}+1\right) r}}{a_{n_{k}^{\prime \prime} r}}
\end{aligned}
$$

and, consequently, from $\left(6.3^{\prime}\right)$ and the convergence of types theorem,

$$
\frac{a_{n_{k}^{\prime} r}}{a_{n_{k}^{\prime \prime} r}} \longrightarrow 1
$$

as $k \rightarrow+\infty$, for every $r$ in $\mathbf{N}$, in contradiction with (6.22). Hence, $\left(a_{r n} / a_{n}\right)_{n}$ converges for every $r$ in $\mathbf{N}$ and, consequently, representation (6.10) turns out to be independent of $\left(n^{\prime}\right)$. Then, from the Prokhorov theorem, $\left(\left(S_{1 n}-\right.\right.$ $\left.\left.c_{n}, S_{2 n}-c_{n}, \ldots\right)\right)_{n}$ converges. Theorem 8 is proved.

Let us conclude with a result on regular variation of $\left(a_{n}\right)_{n}$ in Theorem 8 .

Theorem 9. Under the hypotheses in Theorem $8,\left(a_{n}\right)_{n}$ either varies regularly with exponent $\frac{1}{2}$ or varies regularly with exponent 1 .

P r o of. From the proof of Theorem 8:

$$
\lim _{n \rightarrow+\infty} \frac{a_{r n}}{a_{n}}=\alpha^{(r)}=r \quad \text { or } \quad \sqrt{r}
$$

for every $r$ in $\mathbf{N}$. Then, for any $s$ in $\mathbf{N}$,

$$
\frac{S_{1 r n+s}-b_{r n+s}}{a_{r n+s}}=\frac{S_{1 r n}-b_{r n}}{a_{r n}} \frac{a_{r n}}{a_{r n+s}}+\frac{\sum_{i=r n+1}^{r n+s} X_{i}}{a_{r n+s}}+\frac{b_{r n}-b_{r n+s}}{a_{r n+s}}
$$

and, from the convergence of types theorem,

$$
\lim _{n \rightarrow+\infty} \frac{a_{r n}}{a_{r n+s}}=1, \quad r, s \in \mathbf{N} .
$$

Let $\rho$ be a strictly positive rational and let $r$ be the least integer for which $\rho r \in \mathbf{N}$. Then, for $n=i r(i=1,2, \ldots)$

$$
\frac{a_{[\rho n]}}{a_{n}}=\frac{a_{\rho i r}}{a_{i r}}=\frac{a_{\rho i r}}{a_{i}} \frac{a_{i}}{a_{i r}} .
$$

Hence

$$
\frac{a_{[\rho n]}}{a_{n}} \longrightarrow \alpha^{(\rho)}
$$


and, from (6.23)-(6.24),

$$
\frac{a_{\lfloor\rho(i r+1)]}}{a_{i r+1}}=\frac{a_{\rho i r+[\rho]}}{a_{\rho i r}} \frac{a_{\rho i r}}{a_{i r}} \frac{a_{i r}}{a_{i r+1}} \rightarrow \alpha^{(\rho)}
$$

which entails (6.24) along the sequence: $n=r, r+1,2 r, 2 r+1, \ldots$ Iterating the process a finite number of times, one obtains (6.24) for any strictly positive rational $\rho$. In order to show that (6.24) holds for each $\rho>0$, observe that each subsequence of $\left(a_{[\rho n]}\right)_{n}$ contains an increasing subsequence $\left(a_{\left[\rho n^{\prime}\right]}\right)_{n^{\prime}}$. Then, for any two rationals $\rho_{1}$ and $\rho_{2}$ such that $\rho_{1} \leqslant \rho \leqslant \rho_{2}$,

$$
\alpha^{\left(\rho_{1}\right)} \leqslant \liminf _{n^{\prime \prime}:\left[n^{\prime \prime} \rho\right] \in\left(n^{\prime}\right)} \frac{a_{\left[n^{\prime \prime} \rho\right]}}{a_{n^{\prime \prime}}} \leqslant \limsup _{n^{\prime \prime}:\left[n^{\prime \prime} \rho\right] \in\left(n^{\prime}\right)} \frac{a_{\left[n^{\prime \prime} \rho\right]}}{a_{n^{\prime \prime}}} \leqslant \alpha^{\left(\rho_{2}\right)}
$$

and, in view of the arbitrariness of $\rho_{1}, \rho_{2}$ and the continuity or $\rho \rightarrow \alpha^{(\rho)}$, (6.24) must hold for every $\rho>0$.

\section{Applications}

Now we exhibit some applications of the previous general results to some common situations, analyzed before by several Authors from different viewpoints. In particular, one provides a new deduction of a central limit theorem, for exchangeable random variables without moments, proved by Klass and Teicher [13]. In each case taken into consideration, the corresponding classical result is obtained as corollary of the results explained in Sections 5-6.

7.1. Convergence to the Poisson law. Kendall [10] proved that, if the elements of $\xi^{(n)}$ in (1.3) are 0-1 exchangeable r.v.'s, and if

$$
\lambda_{F_{k n}}(\{0\})=1-\lambda_{F_{k n}}(\{1\})=1-\theta_{n}(\omega)
$$

with

$$
\left\{\begin{array}{l}
n E\left(\theta_{n}\right) \longrightarrow \mu>0 \\
n^{2} E\left(\theta_{n}^{2}\right) \longrightarrow \mu^{2}>0
\end{array}\right.
$$

then the p.d.f. of $S_{1 n}$ converges weakly to the Poisson law with parameter $\mu$.

Kendall'obtained this result by analytical methods. Now, one can prove the same result by showing that (7.1) implies the conditions in Theorem 5 . In fact, given $\varepsilon$ and $\eta>0$,

$$
\begin{aligned}
& P\left(\max _{1 \leqslant i \leqslant n} \lambda_{F_{i n}}\left([-\varepsilon, \varepsilon]^{c}\right)>\eta\right)=P\left(\lambda_{F_{1 n}}\left([-\varepsilon, \varepsilon]^{c}\right)>\eta\right) \\
& \quad \leqslant \frac{1}{\eta} P\left(\left|\xi_{11}^{(n)}\right|>\varepsilon\right) \leqslant \frac{1}{\eta} E\left(\theta_{n}\right) \sim \frac{1}{\eta} \frac{\mu}{n}
\end{aligned}
$$


and this implies $\left(\mathrm{H}_{2}\right)$. Moreover, given $\varepsilon$ and $r$ in $(0,1)$ :

$$
\begin{gathered}
\sum_{k=1}^{n} \int_{|x| \geqslant \varepsilon,|x-1| \geqslant \varepsilon} d F_{k n}(x, \omega)=0, \\
m_{n}(\omega, r)=\sum_{k=1}^{n} \int_{|x|<r} x d F_{k n}(x, \omega)=0, \quad \sigma_{n}^{2}(\omega, r)=0,
\end{gathered}
$$

and

$$
\sum_{k=1}^{n} \int_{|x-1|<\varepsilon} d F_{k n}(x, \omega)=n \theta_{n}(\omega)
$$

Since

$$
P\left(\left|n \theta_{n}(\omega)-\mu\right|>\varepsilon\right) \leqslant \frac{1}{\varepsilon^{2}} E\left(\left(n \theta_{n}-\mu\right)^{2}\right) \longrightarrow 0 \quad \text { (from (7.1)) }
$$

then $\chi_{n}^{(\varepsilon)}$ converges weakly to $\delta_{\mu}$ and, from Theorem $5, S_{1 n} \stackrel{\ell}{\longrightarrow} X$ where $X$ has the Poisson distribution with parameter $\mu$.

In view of Theorem 5 , conditions (7.1) are redundant when it comes to obtaining convergence to the Poisson distribution. In fact, for this purpose, it suffices to replace (7.1) with

$$
\left\{\begin{array}{l}
E\left(\theta_{n}\right)=o(1) \\
n \theta_{n} \stackrel{P}{\longrightarrow} \mu .
\end{array}\right.
$$

Kendall considers the following case in which (7.1) does not hold:

$$
P\left(\theta_{n}=\frac{\mu}{n}\right)=1-\frac{1}{n}, \quad P\left(\theta_{n}=1\right)=\frac{1}{n},
$$

and, nevertheless, the Poisson convergence holds. Indeed, in that very case,

$$
E\left(\theta_{n}\right) \rightarrow 0, \quad P\left(\left|n \theta_{n}-\mu\right|>\varepsilon\right) \leqslant P\left(\theta_{n}=1\right)=\frac{1}{n}
$$

so that we obtain $\left(7: 1^{\prime}\right)$. On the other hand, nothing can be said, without further analysis, about necessity of (7.1') in order that $S_{1 n}$ converges in law to a r.v. distributed according to the Poisson distribution. In fact, (7.1') is necessary in order that $\left(S_{1 n}, S_{2 n}, \ldots\right)$ converges in law to a sequence of independent r.v.'s, distributed according to the Poisson distribution with parameter $\mu$.

7.2. Convergence to the Gaussian law. Consider (1.3) with $\xi_{i j}^{(n)}=$ $X_{i j}^{(n)} / \sqrt{n}(i \geqslant 1 ; 1 \leqslant j \leqslant n)$ and assume that the $X_{i j}^{(n)}$ 's are exchangeable with common conditional p.d.f. $F_{n}(\cdot, \omega)$. Corollary 2 in [18], obtained by martingale methods, states that

$$
\text { if } E\left(X_{i j}^{(n)}\right)=0, \operatorname{Var}\left(\left(X_{i j}^{(n)}\right)^{2}\right)=1 \text { with } E\left(\left(X_{i j}^{(n)}\right)^{4}\right)<+\infty \text {, and }
$$


(a) $E\left(\left(X_{i 1}^{(n)} X_{i 2}^{(n)}\right)^{2}\right) \longrightarrow 1$ as $n \rightarrow+\infty$

(b) $n E\left(X_{i 1}^{(n)} X_{i 2}^{(n)}\right) \rightarrow 0$ as $n \rightarrow+\infty$

(c) $\forall \varepsilon>0, E\left(\left(X_{i 1}^{(n)}\right)^{2} 1_{\left\{\left|X_{i 1}^{(n)}\right|>\varepsilon \sqrt{n}\right\}}\right) \longrightarrow 0$ as $n \rightarrow+\infty$,

then $S_{1 n}$ converges in law to a standard Gaussian r.v.

In fact, this result is an almost direct consequence of Theorem 3 . Indeed, for every $\delta>0$ :

$$
\begin{aligned}
q_{n}^{(\varepsilon)}((\delta,+\infty)) & =P\left(\sum_{k=1}^{n} \lambda_{F_{n}}\left([-\varepsilon \sqrt{n}, \varepsilon \sqrt{n}]^{c}\right)>\delta\right) \\
& \leqslant \frac{n}{\delta} P\left(\left|X_{11}^{(n)}\right|>\varepsilon \sqrt{n}\right) \longrightarrow 0 \quad(\text { from (c)) }
\end{aligned}
$$

and this implies $q_{n}^{(\varepsilon)} \Rightarrow \delta_{0}$. Moreover,

$$
\begin{aligned}
m_{n}(\omega, r) & =\sqrt{n} \int_{\mathbf{R}} x 1_{[0, r]}\left(\frac{|x|}{\sqrt{n}}\right) d F_{n}(x, \omega) \\
& =\sqrt{n}\left\{\int_{\mathbf{R}} x d F_{n}(x, \omega)-\int_{\mathbf{R}} x 1_{(r,+\infty)}\left(\frac{|x|}{\sqrt{n}}\right) d F_{n}(x, \omega)\right\}
\end{aligned}
$$

and

$$
\begin{aligned}
P\left(\left|\sqrt{n} \int_{\mathbf{R}} x d F_{n}(x, \omega)\right|>\delta\right) & \leqslant \frac{n}{\delta^{2}} E\left(X_{11}^{(n)} X_{12}^{(n)}\right) \rightarrow 0 \quad \text { (from (b)); } \\
\left|\sqrt{n} \int_{\mathbf{R}} x 1_{(r,+\infty)}\left(\frac{|x|}{\sqrt{n}}\right) d F_{n}(x, \omega)\right| & \leqslant \frac{1}{r} \int_{\mathbf{R}}|x|^{2} 1_{(r \sqrt{n},+\infty)}(|x|) d F_{n}(x, \omega) .
\end{aligned}
$$

One derives from (c),

$$
\begin{aligned}
& P\left(\int_{\mathbf{R}}|x|^{2} 1_{(r \sqrt{n},+\infty)}(|x|) d F_{n}(x, \omega)>\delta\right) \\
& \quad \leqslant \frac{1}{\delta} E\left(\left|X_{11}^{(n)}\right|^{2} 1_{(r \sqrt{n},+\infty)}\left(\left|X_{11}^{(n)}\right|\right)\right) \longrightarrow 0 .
\end{aligned}
$$

Hence, $m_{n}(\omega, r)$ converges in law to a r.v. which degenerates at 0 , and

$$
\begin{aligned}
\sigma_{n}^{2}(\omega, r)= & \left\{\int_{\mathbf{R}} \frac{|x|^{2}}{n} \mathbf{1}_{[0, r]}\left(\frac{|x|}{\sqrt{n}}\right) d F_{n}(x, \omega)\right. \\
& \left.-\left(\int_{\mathbf{R}} \frac{x}{\sqrt{n}} \mathbf{1}_{[0, r]}(|x| / \sqrt{n}) d F_{n}(x, \omega)\right)^{2}\right\} \\
= & \int_{\mathbf{R}}|x|^{2} d F_{n}(x, \omega)-\int_{\mathbf{R}}|x|^{2} 1_{(r \sqrt{n},+\infty)}(|x|) d F_{n}(x, \omega) \\
& -\left\{\int_{\mathbf{R}} x d F_{n}(x, \omega)-\int_{\mathbf{R}} x 1_{(r \sqrt{n},+\infty)}(|x|) d F_{n}(x, \omega)\right\}^{2} .
\end{aligned}
$$


But

$$
\begin{aligned}
& \lim _{n \rightarrow+\infty} E\left(\int_{\mathbf{R}}|x|^{2} 1_{(r \sqrt{n},+\infty)}(|x|) d F_{n}(x, \omega)\right)=0 \\
& E\left(\left\{\int_{\mathbf{R}} x d F_{n}(x, \omega)\right\}^{2}\right)=E\left(X_{11}^{(n)} X_{12}^{(n)}\right)=o\left(\frac{1}{n}\right) \\
& E\left(\left\{\int_{\mathbf{R}} x 1_{(r \sqrt{n},+\infty)}(|x|) d F_{n}(x, \omega)\right\}^{2}\right) \\
& \quad=E\left(X_{11}^{(n)} X_{12}^{(n)} 1_{(r \sqrt{n},+\infty)}\left(\left|X_{11}^{(n)}\right|\right) 1_{(r \sqrt{n},+\infty)}\left(\left|X_{12}^{(n)}\right|\right)\right) \\
& \quad \leqslant E\left(\left(X_{11}^{(n)}\right)^{2} 1_{(r \sqrt{n},+\infty)}\left(\left|X_{11}^{(n)}\right|\right)\right) \rightarrow 0 \quad \text { (from (c)) }
\end{aligned}
$$

and

$$
\begin{aligned}
& E\left(\left|\int_{\mathbf{R}} x d F_{n}(x, \omega) \int_{\mathbf{R}} x 1_{(r \sqrt{n},+\infty)}(|x|) d F_{n}(x, \omega)\right|\right) \\
& \left.\quad \leqslant\left\{E\left(\left|X_{11}^{(n)}\right|^{2} 1_{(r \sqrt{n},+\infty)}\left(\left|X_{11}^{(n)}\right|\right)\right)\right\}^{1 / 2} \rightarrow 0 \quad \text { (from }(\mathrm{c})\right)
\end{aligned}
$$

Finally,

$$
\begin{aligned}
& P\left(\left|\int_{\mathbf{R}}\left(x^{2}-1\right) d F_{n}(x, \omega)\right|>\delta\right) \\
& \quad \leqslant \frac{1}{\delta^{2}}\left\{E\left(\left|X_{11}^{(n)} X_{12}^{(n)}\right|^{2}\right)-1\right\} \rightarrow 0 \quad \text { (from (a)) }
\end{aligned}
$$

and, consequently, $\sigma_{n}^{2}(\omega, r) \stackrel{P}{\longrightarrow} 1$. Then, the thesis follows from Theorem 3 with $\pi=\delta_{0} \otimes \delta_{1}$.

7.3. Convergence to a scale mixture of Gaussian laws. Eaton, Fortini and Regazzini 5 deal with the problem of the convergence of $\left(S_{1 n}-\right.$ $\left.c_{n}\right)_{n}$ when, besides (6.1)-(6.3) with $c_{n}=0, E\left(X_{11}\right)=0$ and $E\left(X_{11}^{2}\right)<+\infty$ hold.

Under these conditions, either: $a_{n} \sim c \sqrt{n}$ and

$$
\begin{gathered}
\lim _{n \rightarrow+\infty} E\left(\operatorname { e x p } \left\{i \sum_{\substack{k=1 \\
\left(t_{1}, \ldots, t_{m}\right) \in \mathbf{R}^{m}, m=1,2, \ldots}} \prod_{(0,+\infty)} \prod_{k=1}^{m} \exp \left\{-\frac{1}{2} t_{k}^{2} b\right\} \pi_{1}(d b)\right.\right. \\
\end{gathered}
$$

or $a_{n} \sim c n$ and

$$
\begin{gathered}
\lim _{n \rightarrow+\infty} E\left(\exp \left\{i \sum_{k=1}^{n} t_{k} S_{k n}\right\}\right) \\
\left(t_{1}, \ldots, t_{m}\right) \in \int_{[0,+\infty)} \prod_{k=1}^{m} \exp \left\{i t_{k} a\right\} \gamma(d a) \\
m=1,2, \ldots
\end{gathered}
$$


This result can be deduced from Theorem 3 and Corollary 1 , as follows. If $E\left(X_{11} X_{12}\right)=0$, then

$$
\begin{aligned}
E\left(\exp \left\{i \frac{t}{\sqrt{n}} \sum_{j=1}^{n} X_{1 j}\right\}\right) & =E\left(H\left(\frac{t}{\sqrt{n}}, \omega\right)^{n}\right) \\
& \rightarrow E\left(\exp \left\{-\frac{t^{2}}{2} \sigma^{2}(\omega)\right\}\right)
\end{aligned}
$$

where $H$ is the Fourier-Stieltjes transform of the conditional p.d.f. $F$ of $X_{11}$, in de Finetti's representation, and $\sigma^{2}(\omega)$ represents the conditional variance. In view of $(6.3), P\left(\sigma^{2}(\omega)=0\right)<1$. Indeed, if $P\left(\sigma^{2}(\omega)=0\right)=1$, then $P\left(X_{11}=0\right)=1$ and this would imply that $S_{1 n}$ converges in probability to 0 , in contradiction with (6.3). Then, from (6.3), (7.4) and the convergence of types theorem,

$$
\frac{a_{n}}{\sqrt{n}} \longrightarrow c>0 \text {. }
$$

At this point, (7.2) follows from Theorem 3. In fact, $\left(\mathrm{H}_{1}\right)$ holds and for every $\delta>0$ and $r>0$ :

$$
\begin{aligned}
q_{n}^{(\varepsilon)}((\delta,+\infty)) & \leqslant \frac{n}{\delta} P\left(\left|X_{11}\right|>\varepsilon a_{n}\right) \\
& \leqslant \frac{n}{\varepsilon^{2} \delta a_{n}^{2}} E\left(\left|X_{11}\right|^{2} 1_{\left\{\left|X_{11}\right|>\varepsilon a_{n}\right\}}\right) \rightarrow 0, \\
\left|m_{n}(\omega, r)\right| & =\left|\frac{n}{a_{n}} \int_{\mathbf{R}} x 1_{[0, r]}\left(\frac{|x|}{a_{n}}\right) d F(x, \omega)\right| \\
& =\left|\frac{n}{a_{n}} \int_{\mathbf{R}} x 1_{(r,+\infty)}\left(\frac{|x|}{a_{n}}\right) d F(x, \omega)\right| \\
& \leqslant \frac{n}{r a_{n}^{2}} \int_{\mathbf{R}}|x|^{2} \mathbf{1}_{\left(r a_{n},+\infty\right)}(|x|) d F(x, \omega)
\end{aligned}
$$

and

$$
\begin{aligned}
& P\left(\int_{\mathbf{R}}|x|^{2} 1_{\left(r a_{n},+\infty\right)}(|x|) d F(x, \omega)>\delta\right) \\
& \leqslant \frac{1}{\delta} E\left(\left(X_{11}^{2}\right) 1_{\left(r a_{n},+\infty\right)}\left(\left|X_{11}\right|\right)\right) \rightarrow 0 \\
\sigma^{2}(\omega, r)= & \frac{n}{a_{n}^{2}}\left\{\int_{|x|<r a_{n}} x^{2} d F(x, \omega)-\left[\int_{|x|<r a_{n}} x d F(x, \omega)\right]^{2}\right\} \\
\sim & \frac{n}{a_{n}^{2}} \int_{|x|<r a_{n}} x^{2} d F(x, \omega)
\end{aligned}
$$

which converges to $\sigma^{2}(\omega) / c^{2}, P$-a.s. This implies (5.2) with $\pi=\delta_{0} \otimes \pi_{1}$ and, consequently, (7.2). To complete the proof, one has to consider the case in which $E\left(X_{11} X_{12}\right) \neq 0$. Since $E\left(X_{11}\right)$ is finite, the strong law of large 
numbers, for sequences of exchangeable r.v.'s, implies that a r.v. $\alpha$ exists such that

$$
\frac{1}{n} \sum_{j=1}^{n} X_{1 j} \rightarrow \alpha \quad(P \text {-a.s. })
$$

Moreover $\dot{\alpha}$ is nondegenerate. In fact, if $P(\alpha=c)=1$ for some suitable constant $c$, then $c=0$ (since $E\left(X_{11}\right)=0$ ) and, consequently, $E\left(X_{11} X_{12}\right)=0$, in contradiction with the assumption. At this point, (6.3) and the convergence of types theorem imply

$$
\frac{a_{n}}{n} \rightarrow c>0
$$

Now, (7.3) follows from Corollary 1. Indeed,

$$
\sigma_{n}^{2}(\omega, r) \stackrel{P}{\longrightarrow} 0, \quad \sum_{k=1}^{n} \int_{|x| \geqslant \varepsilon a_{n}} d F(x, \omega) \stackrel{P}{\longrightarrow} 0
$$

follow from (7.5)-(7.7). Moreover,

$$
m_{n}(\omega, r)=\frac{n}{a_{n}} \int_{\mathbf{R}} x 1_{\left[0, r a_{n}\right]}(|x|) d F(x, \omega) \rightarrow \frac{1}{c} \alpha(\omega) \quad(P \text {-a.s. })
$$

7.4. Convergence to the Gaussian law. Exchangeable summands without moments. Klass and Teicher [13] provide necessary and sufficient conditions in order that $\left(S_{1 n}-c_{n}\right)_{n}$ converges in law to a standard Gaussian r.v. $Z$, under assumptions (6.1)-(6.2). From Theorem 4 and Corollary 1, via (6.19)-(6.21) one immediately obtains the following theorem, which implies the basic Klass and Teicher central limit problem.

If $\left(S_{1 n}-c_{n}\right)_{n}$ converges in law to $Z$, and if $F=F(\cdot, \omega)$ denotes the conditional p.d.f. of $X_{11}$ in de Finetti's representation, then

$$
n \lambda_{F}\left(\left[-\varepsilon a_{n}, \varepsilon a_{n}\right]^{c}\right) \stackrel{P}{\longrightarrow} 0, \quad \varepsilon>0
$$

and either

$$
\left\{\begin{array}{l}
\frac{a_{r n}}{a_{n}} \rightarrow \sqrt{r} \\
\frac{n}{a_{n}} \int_{|x|<\tau} x d F(x, \omega)-\frac{b_{n}}{a_{n}} \stackrel{P}{\longrightarrow} 0, \\
\frac{n}{a_{n}^{2}}\left\{\int_{|x|<\tau} x^{2} d F(x, \omega)-\left(\int_{|x|<\tau} x d F(x, \omega)\right)^{2}\right\} \stackrel{P}{\longrightarrow} 1
\end{array}\right.
$$


(for some $\tau>0$ ) or

$$
\left\{\begin{array}{l}
\frac{a_{r n}}{a_{n}} \rightarrow r \\
\frac{n}{a_{n}} \int_{|x|<\tau} x d F(x, \omega)-\frac{b_{n}}{a_{n}} \stackrel{\ell}{\longrightarrow} Z, \\
\frac{n}{a_{n}^{2}}\left\{\int_{|x|<\tau} x^{2} d F(x, \omega)-\left(\int_{|x|<\tau} x d F(x, \omega)\right)^{2}\right\} \stackrel{P}{\rightarrow} 0
\end{array}\right.
$$

(for some $\tau>0$ ). Conversely, if there exist sequences $\left(b_{n}\right)_{n}$ and $\left(a_{n}\right)_{n}$, $0<a_{n} \rightarrow+\infty$, such that (7.8) and either (7.9) or (7.10) hold, then $\left(S_{1 n}-c_{n}\right)_{n}$ converges in law to $Z$.

As a matter of fact, the above conditions for convergence to $Z$ formally differ from the ones in Theorem 2 in [13], but they are obviously equivalent. In any case, one can use Theorem 4 and Corollary 1 to provide an alternative proof of Theorem 2 in [13]. Indeed, if $S_{1 n}-c_{n} \stackrel{\ell}{\rightarrow} Z$, from Theorem 1 in [13] it follows that

$$
T_{1 n}-c_{n}:=\frac{1}{a_{n}} \sum_{i=1}^{n} X_{1 i} 1_{\left\{\left|X_{1 i}\right|<\varepsilon_{n} a_{n}\right\}}
$$

converges in law to $Z$ for a suitable sequence $\varepsilon_{n} \downarrow 0$, and condition (29) in [13] follows from Lemma 1 in [13]. In fact, since $\left(S_{1 n}-c_{n}, S_{2 n}-c_{n}, \ldots\right)$ converges in law and $P\left(\left\{S_{1 n} \neq T_{1 n}\right\} \cup \cdots \cup\left\{S_{k n} \neq T_{k n}\right\}\right) \longrightarrow 0$, for every $k$, then $\left(T_{1 n}-c_{n}, T_{2 n}-c_{n}, \ldots\right)$ converges in law to the same limit of $\left(S_{1 n}-\right.$ $\left.c_{n}, S_{2 n}-c_{n}, \ldots\right)$. Hence, if $a_{r n} / a_{n} \longrightarrow \sqrt{r}$, then (6.20) and (6.21) state that such a limit is a mixture of Gaussian laws and, from Theorem 4 applied to $\left(T_{1 n}-c_{n}, T_{2 n}-c_{n}, \ldots\right)$ one deduces (30) in [13]. Alternatively, if $a_{r n} / a_{n} \longrightarrow r$, then (6.19) and (6.21) state that the limit of $\left(T_{1 n}-c_{n}, T_{2 n}-c_{n}, \ldots\right)$ is a mixture of degenerate laws and (31) in [13] directly follows from Corollary 1. Conversely, if (29) and (30) [(29) and (31)] in [13] hold, then convergence in law of $\left(T_{1 n}-c_{n}\right)$ to $Z$ directly follows from Theorem 4 (Corollary 1 ). Moreover, from (29) one easily deduces that $S_{1 n}-c_{n} \stackrel{\ell}{\longrightarrow} Z$.

Acknowledgments. We should like to thank Professor V. V. Sazonov for his comments and his improvements in the proofs in Section 3.

\section{REFERENCES}

1. Billingsley $P$. Convergence of Probability Measures. - New York: Wiley, 1968.

2. Billingsley $P$. Weak Convergence of Measures: Applications in Probability. Philadelphia: SIAM, 1971.

3. Doob J. L. Measure Theory. - New York: Springer-Verlag, 1994.

4. Eagleson G. K. Weak limit theorems for exchangeable random variables. - In: Exchangeability in Probability and Statistics. Ed. by G. Koch and F. Spizzichino. Amsterdam: North-Holland, 1982, p. 251-268. 
5. Eaton M. L., Fortini S., Regazzini E. Spherical symmetry: an elementary justification. - J. Italian Statist. Soc., 1993, v. 2, p. 1-16.

6. De Finetti $B$. Sulle funzioni ad incremento aleatorio. - Rend. R. Accad. Naz. Lincei Cl. Sci. Fis. Mat. Nat. (S. $6^{\mathrm{a}}$ ), 1929, v. 10, p. 163-168.

7. De Finetti $B$. Le funzioni caratteristiche di legge istantanea. - Rend. R. Accad. Naz. Lincei Cl. Sci. Fis. Mat. Nat. (S. $6^{a}$ ), 1930, v. 12, p. 278-282.

8. De Finetti $B$. Sur la condition d'équivalence partielle. - Actualités Scientifique et Industrielles, № 739. Paris: Hermann, 1938.

9. Folland G. B. Real Analysis: Modern Techniques and their Applications. - New York: Wiley, 1984.

10. Kendall $D$. G. On finite and infinite sequences of exchangeable events. - Studia Sci. Math. Hungar., 1967, v. 2, p. 319-327.

11. Хикчия $А$. Я. Новый вид одной формулы П. Леви. - Бюллетень Московского ун-та, секц. А, 1937 , т. 1 , в. 1, с. 1-15.

12. Kolmogorov A. N. Sulla forma generale di un processo stocastico omogeneo (un problema di Bruno de Finetti). - Rend. R. Accad. Naz. Lincei Cl. Sci. Fis. Mat. Nat. (S. $\left.6^{\mathrm{a}}\right), 1932$, v. 15 , p. $805-808$, p. $866-869$.

13. Klass $M$., Teicher $H$. The central limit theorem for exchangeable random variables without moments. - Ann. Probab., 1987, v. 15, p. 138-153.

14. Klopotowski A. Mixtures of infinitely divisible distributions as limit laws for sums of dependent random variables. $-Z$. Wahrscheinlichkeitstheor. verw. Geb., 1980, v. 51, p. 101-113.

15. Lévy $P$. Sur les intégrales dont les éléments sont des variables aléatoires indépendentes. - Annali R: Scuola Norm. Sup. Pisa, 1934, v. 3, p. 337-366; 1935, v. 4, p. 217-218.

16. Loève $M$. Probability Theory, I. - New York: Springer-Verlag, 1977.

17. Taylor R. L., Daffer P. Z., Patterson R. F. Limit Theorems for Sums of Exchangeable Random Variables. Rowman \& Allanheld Publ., 1985.

18. Weber $N$. C. A martingale approach to central limit theorems for exchangeable random variables. - J. Appl. Probab., 1980, v. 17, p. 662-673.

19. Xue Xing-Hong. On the principle of conditioning and convergence to mixtures of distributions for sums of dependent random variables. - Stoch. Process. Appl., 1991, v. 37 , p. $175-186$.

20. Aldous D. J. Exchangeability and related topics. - Lect. Notes Math., 1985, v. 1117, p. 1-198.

21. Regazzini $E$. Coherence, exchangeability and statistical models (de Finetti's stances «revisited»). - Atti del Convegno «Sviluppi metodologici nei diversi approcci all'inferenza statistica», Pitagora, Bologna, 1991, v. 2, p. 101-137.

Поступила в редакцию

30.IX.1994

Исправленный вариант

25.XII.1995 